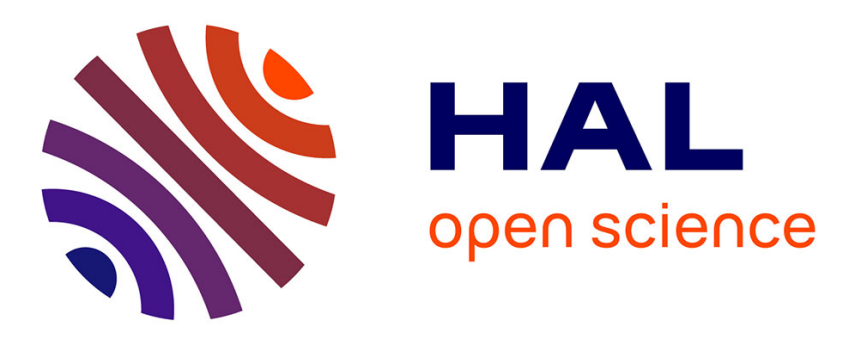

\title{
Pitch Axis Control for a Guided Projectile in a Wind Tunnel-based Hardware-in-The-Loop Setup
}

Guillaume Strub, Spilios Theodoulis, Vincent Gassmann, Simona Dobre, Michel Basset

\section{- To cite this version:}

Guillaume Strub, Spilios Theodoulis, Vincent Gassmann, Simona Dobre, Michel Basset. Pitch Axis Control for a Guided Projectile in a Wind Tunnel-based Hardware-in-The-Loop Setup. AIAA Journal of Spacecraft and Rockets, 2015, 10.2514/6.2015-0153 . hal-01289585

\section{HAL Id: hal-01289585 \\ https://hal.science/hal-01289585}

Submitted on 17 Mar 2016

HAL is a multi-disciplinary open access archive for the deposit and dissemination of scientific research documents, whether they are published or not. The documents may come from teaching and research institutions in France or abroad, or from public or private research centers.
L'archive ouverte pluridisciplinaire HAL, est destinée au dépôt et à la diffusion de documents scientifiques de niveau recherche, publiés ou non, émanant des établissements d'enseignement et de recherche français ou étrangers, des laboratoires publics ou privés. 


\title{
Pitch Axis Control for a Guided Projectile in a Wind
}

\section{Tunnel-based Hardware-In-the-Loop Setup}

\author{
Guillaume Strub ${ }^{1}$, \\ French-German Research Institute of Saint-Louis (ISL), 68301 Saint-Louis, France and \\ Laboratoire MIPS EA2332, Université de Haute-Alsace, 68093 Mulhouse, France \\ Spilios Theodoulis ${ }^{2}$, Vincent Gassmann ${ }^{3}$, Simona Dobre ${ }^{4}$ \\ French-German Research Institute of Saint-Louis (ISL), 68301 Saint-Louis, France \\ Michel Basset ${ }^{5}$ \\ Laboratoire MIPS EA2332, Université de Haute-Alsace, 68093 Mulhouse, France
}

This article details the design of a pitch axis autopilot for an $80 \mathrm{~mm}$ fin-stabilized, canard-guided projectile and its validation on a Hardware-In-the-Loop test setup. This setup is built around an autonomous projectile prototype, which is installed in the test section of a wind tunnel by the means of a 3-DoF gimbaled structure. The autopilot design is based on a family of linearized dynamic models of the projectile, whose parameters were estimated from experimental data. Several control approaches are considered. Using the $\mathcal{H}_{\infty}$ robust control framework, a full-order disturbance rejection controller is designed, taking into account the limited actuator dynamics. A fixed-order, fixed-structure controller of lower complexity with the same performance objective is also designed, using a nonsmooth $\mathcal{H}_{\infty}$ technique. The tracking performance of these controllers is improved with the addition of a feedforward controller. A final approach considers the disturbance rejection and reference tracking as a multi-objective problem, where the feedback and the feedforward controllers are designed in a single step. The performance of these approaches is then assessed and compared using numerical simulation as well as experimental results gathered from the Hardware-In-the-Loop setup.

\footnotetext{
${ }^{1}$ PhD Student, Guidance, Navigation and Control Department; guillaume.strub@isl.eu. AIAA Student Member

2 Research Associate, Guidance, Navigation and Control Department; spilios.theodoulis@isl.eu. AIAA Associate Member

${ }^{3}$ Research Associate, Guidance, Navigation and Control Department; vincent.gassmann@isl.eu.

${ }^{4}$ Research Associate, Aerodynamics and Exterior Ballistics; simona.dobre@isl.eu. AIAA Associate Member

5 Professor, Control Department, michel.basset@uha.fr
} 


\section{Nomenclature}

Time-varying signals

$\begin{array}{ll}\alpha_{r}, \alpha & \text { Reference and measured angle of attack, deg } \\ d & \text { Disturbance signal } \\ \delta_{u}, \delta_{m} & \text { Reference and measured pitch control deflection, deg } \\ p, q, r & \text { Body angular rates, deg/s } \\ \phi, \theta, \psi & \text { Euler angles (roll, pitch and yaw), deg }\end{array}$

\section{Parameters}

$h \quad$ Projectile altitude, $\mathrm{m}$

$M_{\alpha d}, M_{q d} \quad$ Body disturbance model parameters

$k_{d}, k_{\delta_{r}} \quad$ Input scaling factors

$k_{q} \quad$ Rate damping gain

$M_{q \alpha}, M_{q q}, M_{q \delta} \quad$ Body dynamic model parameters

$\omega_{0}, \zeta \quad$ Reference model bandwidth, rad/s, and damping ratio

$\rho \quad$ Operating point

$\boldsymbol{\theta} \quad$ Parameter vector

V Airspeed, $\mathrm{m} / \mathrm{s}$

Models and subsystems

$\boldsymbol{G} \quad$ Open-loop plant

$\boldsymbol{G}_{\mathrm{A}}, \boldsymbol{G}_{\mathrm{S}} \quad$ Actuator and sensor models

$\boldsymbol{G}_{\mathrm{B}}, \boldsymbol{H}_{\mathrm{B}} \quad$ Airframe dynamic and disturbance models

$\boldsymbol{G}_{\mathrm{P}} \quad$ Rate-damped plant

$\boldsymbol{K}_{\mathrm{FB}} \quad$ Feedback (disturbance rejection) controller

$\boldsymbol{K}_{\mathrm{FF}}, \boldsymbol{K}_{\mathrm{FF}}^{\prime} \quad$ Feedforward (reference tracking) controller, filter and injection forms

$\boldsymbol{T}_{\text {ref }} \quad$ Reference model

Synthesis-related symbols 


$\begin{array}{ll}k_{i, L F}, k_{i, H F}, \omega_{i} & \text { Weighting filter-related low- and high-frequency gains and }-3 \mathrm{~dB} \text { bandwidth } \\ \boldsymbol{P} & \text { Augmented plant } \\ r, e, e_{M M} & \text { Reference input, tracking error and model-matching error } \\ \boldsymbol{S}_{i \rightarrow e} & \text { Closed-loop sensitivity function to disturbance channel } i \\ \boldsymbol{T}_{i \rightarrow j} & \text { Transfer fonction from channels } i \text { to } j \\ u, v & \text { Controller output and input } \\ \boldsymbol{W}_{i} & \text { Weighting filter } \\ w_{i}, z_{i} & \text { Exogenous inputs and performance outputs } \\ \text { Robustness analysis-related symbols }_{\Delta_{I}} & \text { Complex perturbation transfer function } \\ \ell_{I} & \text { Uncertainty radius } \\ \boldsymbol{G}_{\mathrm{P}}, \overline{\boldsymbol{G}}_{\mathbf{P}} & \text { Perturbed and nominal body pitch rate dynamics model } \\ w_{I} & \text { Multiplicative uncertainty weight }\end{array}$

\section{Introduction}

An important trend in the field of guided ammunition research is the development of low-cost gunlaunched guided projectiles that aim at overcoming the limitations of traditional artillery ammunition (e.g. in terms of accuracy, range, collateral damage and round expenditure). The development process for such weapons is based on the traditional missile design procedure, which can be long and costly. As the design of the control laws is usually done using model-based techniques, an accurate model of the projectile's dynamic behavior is crucial. The parameters of such models are traditionally quantified using various techniques such as semi-empirical aerodynamic prediction codes, wind tunnel testing, Computational Fluid Dynamics (CFD) predictions and/or free-flight tests. However, these techniques may lack precision for some airframe configurations or flight conditions and are usually expensive in terms of time and cost.

Experimental validation and testing of the designed control laws is usually done through free-flight tests or Hardware-In-the-Loop simulations on flight tables. The former technique requires a gun-hardened readyto-fly prototype and a sufficiently mature controller design, therefore it is unsuitable for initial testing. The 
latter technique consists in using a functional prototype with a 3-DoF actuation system and simulating the aerodynamic environment using a model. Although they are much more flexible and less expensive than free-flight tests, such simulations are limited in accuracy regarding the free-flight dynamic behavior.

The present paper proposes a novel, low-cost technique applicable to parameter estimation and control algorithm validation for guided projectiles, trying to overcome the shortcomings associated with the above two techniques. To this end, a fully functional guided projectile prototype based on low-cost, off-the-shelf hardware is installed in the test section of a wind tunnel by the means of a 3-DoF gimbaled support structure, enabling rotation on the roll, pitch and yaw axes. This setup, illustrated in Figure 1 and referred to as ACHILES (Automatic Control Hardware-In-the-Loop Experimental Setup), provides with realistic flight conditions in a controlled experimental environment and enables rapid algorithm development and testing through the use of industry-standard software.

To the author's knowledge, there are very few similar setups targeted at identification and control studies for guided projectiles and similar systems. In Hann et al. [1], the parameters of a sounding rocket roll dynamics model are estimated from experimental data gathered using an actual prototype in a vertical wind tunnel. Fresconi [2] proposes a guided projectile prototype similar to our proposal, with an emphasis on the use of low-cost, off-the-shelf components and their resistance to high accelerations. Using aerodynamic data from PRODAS studies, a linear quadratic optimal roll controller is designed and tested on the projectile prototype in a wind tunnel. In both cases, the experimental setups in these studies only allows motion on the roll axis, whereas ACHILES allows the three angular degrees of freedom. In Patel et al. [3], a missile flow-control-based yaw actuator is devised. A closed-loop controller modulates flow effectors to produce a yawing moment by exploiting flow asymmetry around the missile forebody. Dynamic tests in a wind tunnel demonstrated the ability of the device to generate and maintain a range of yawing moments during high-incidence pitch sweeps.

Previous work by the authors [4] focused on the identification of ACHILES' pitch axis dynamics. Using flight mechanics principles, a nonlinear pitch axis model is constructed, then linearized around a family of equilibrium points. The projectile is then stimulated around each operating point and the model parameters are estimated using the collected data, resulting in a family of linear models. Results have shown that parameters do not vary significantly along the flight envelope for a fixed airspeed, allowing a single-model 


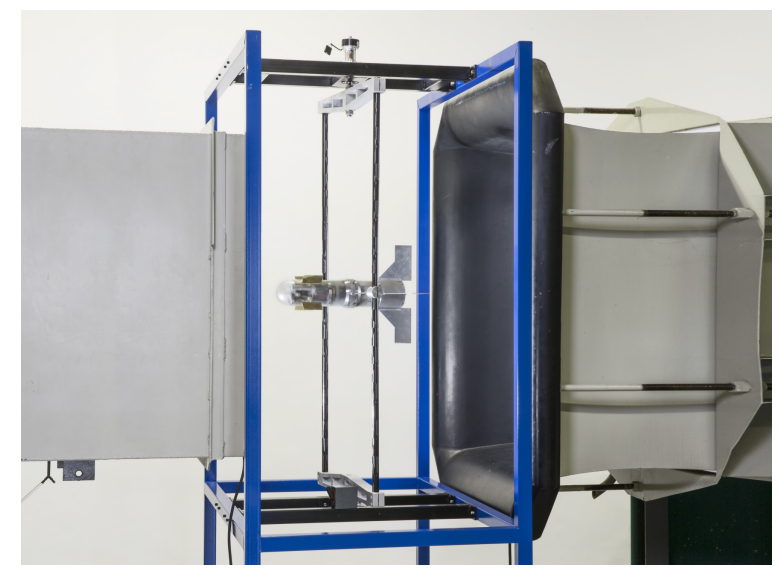

Fig. 1 Overview of the ACHILES Hardware-In-the-Loop experimental setup, including the guided projectile prototype, the 3-DoF support structure and the wind tunnel.

uncertain representation of the pitch dynamics.

In this paper, this knowledge is leveraged so as to design a pitch axis autopilot. The main purpose of this control system is twofold: it has to ensure good tracking of the reference signal while minimizing the effects of disturbances on the system output. The $\mathcal{H}_{\infty}$ framework has been used successfully for missile flight control design [5-7], which share several characteristics with the ACHILES projectile prototype. These techniques are well-suited to this task, as they take into account limiting factors, e.g. modeling uncertainty, operating condition variations, external disturbances or limited actuator and sensor bandwidth, while guaranteeing system stability and the desired performance. Moreover, recent advances in $\mathcal{H}_{\infty}$ control, such as nonsmooth $\mathcal{H}_{\infty}$ synthesis [8] and multi-objective control $[9,10]$, enable the design of low-complexity, high-performance autopilots. As it is desirable to have a low-order control system, three design approaches with decreasing complexity are proposed and compared using numerical simulation as well as Hardware-In-the-Loop experiments.

This paper is organized as follows: Section II presents the hardware and software components of the ACHILES experimental setup. In Section III, an uncertain model of the body dynamics is built using estimated models, and the actuator model is presented. Section IV details the design of the pitch autopilot, using three approaches with decreasing controller complexity. The robustness of the designed control laws is assessed in Section V and in Section VI the proposed controllers are implemented and compared on the projectile prototype. 


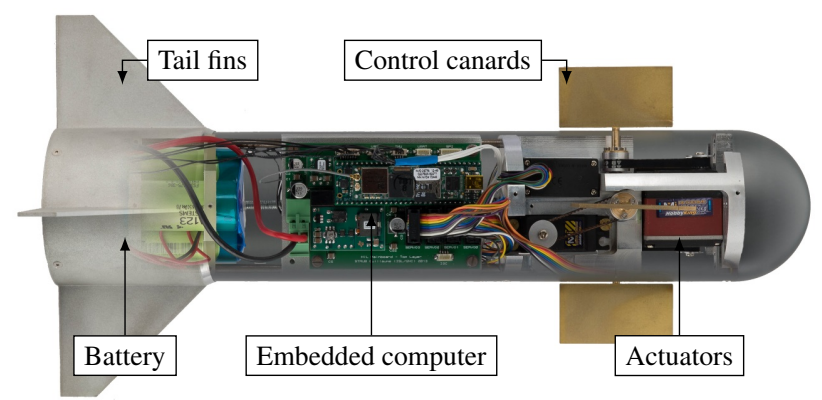

Fig. 2 Inside view of the ACHILES projectile prototype. The IMU, which is on the other side of the main electronics boar, is not pictured here.

\section{Experimental Setup}

The ACHILES projectile prototype is based on a fin-stabilized $80 \mathrm{~mm}$ caliber shell with four nose-located canards, of which an inside view is given in Fig. 2. This missile-like structure has several advantages for identification and control investigations: as the frame is stabilized with tail fins, the stability only depends on the geometry of the aerodynamic surfaces, as opposed to a gyrostabilized projectile. Moreover, the crossaxis coupling as well as the mechanical complexity are greatly reduced since there is no need for a high spin rate. The projectile is installed in the test section of a subsonic wind tunnel by the means of a 3-DoF gimbaled structure as shown in Fig. 1. This structure enables rotation along the roll, pitch and yaw axes while restraining all linear motion.

The projectile is controlled using aerodynamic surfaces (canards), which provide good control authority. The canards are independently driven by modified COTS R/C servomotors, which have been retrofitted with a custom control board in order to improve their linearity and dynamic behavior. A Microstrain 3DM-GX3 Inertial Measurement Unit (IMU) provides the control algorithms with an estimate of the projectile's angular attitude and velocities, and is based on a set of three accelerometers, magnetometers and gyrometers and an embedded sensor fusion algorithm.

In order to speed up algorithm development, a rapid prototyping environment based on MATLAB/Simulink has been developed. The algorithms are designed as Simulink diagrams, using standard blocks as well as custom blocks for interacting with the projectile's actuators and sensors. These diagrams are then converted to $\mathrm{C}$ code and compiled using the MATLAB Coder and Simulink Coder toolboxes. The resulting software is executed onboard the projectile, which features a realtime Linux distribution running on 


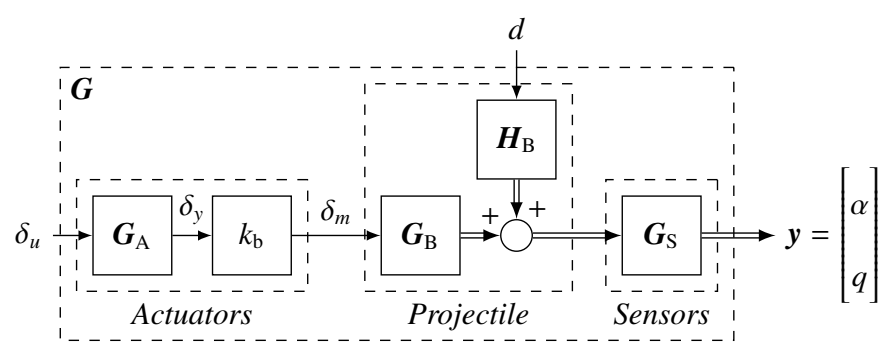

Fig. 3 Open-Loop plant model $G$.

an ARM-based Gumstix embedded computer. A more detailed review of the hardware components and the software stack is given in Ref. [4].

\section{Modeling}

The open-loop pitch dynamics $\boldsymbol{G}$ of the ACHILES prototype can be represented as a series interconnection of the actuator model, the body model and the sensors model, as highlighted in Fig. 3. These models are detailed in the following paragraphs.

\section{A. Actuators}

The ACHILES actuators are based on Hobbyking HK47010 metal-geared servomotors, where the stock control board has been replaced with a custom microcontroller-based control board and an AS5045 12-bit magnetic absolute position sensor. The servos are linked to the control fins using a belt drive with reduction ratio $k_{\mathrm{b}}=16 / 18$; the belt dynamics are not considered here for simplicity. As a complete control study has been conducted on the actuators, a model of their dynamic behavior can be built using this knowledge [11]. To this end, the complete servo model is represented as a block diagram in Fig. 4 and the corresponding transfer function is

$$
\boldsymbol{G}_{\mathrm{A}}=\frac{k_{\mathrm{pos}} K\left(k_{\mathrm{p}} s+k_{\mathrm{i}}\right)}{\tau s^{3}+\left(1+K k_{\mathrm{p}}\right) s^{2}+K\left(k_{\mathrm{i}}+k_{\mathrm{pos}} k_{\mathrm{p}}\right) s+K k_{\mathrm{pos}} k_{\mathrm{i}}}
$$

where the parameter values are given in Table 3 (see Appendix).

The open-loop servo dynamics are represented with a first-order model $K /(1+\tau s)$, where $\tau$ captures the mechanical time constant of the motor and the gear train. The implemented control system consists in a cascade of a proportional position controller $k_{p o s}$ and a proportional-integral velocity controller $k_{p}+k_{i} / s$. These parameters have been tuned using a nonsmooth $\mathcal{H}_{\infty}$ mixed-sensitivity design procedure [8], which 


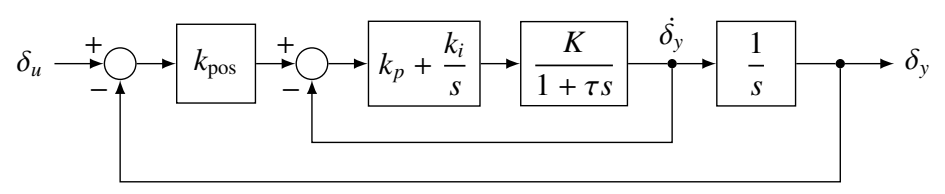

Fig. 4 Servo controller structure.

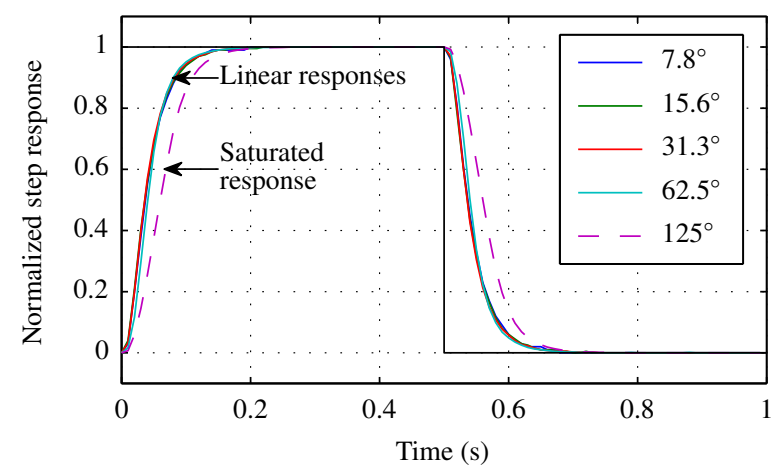

Fig. 5 Normalized actuator step responses for a set of reference angles.

matches the closed-loop response to a second-order system $\boldsymbol{T}_{\text {ref }}^{\mathrm{A}}$ with bandwidth $\omega_{0}=50 \mathrm{rad} / \mathrm{s}$ and an optimal damping $\zeta=0.78$. As an additional constraint, the motor control voltage must remain under the supply voltage at all times, for any reference step up to $\pm 60^{\circ}$ in order to avoid saturation.

This controller is implemented on the custom servo control boards with the addition of a simple antiwindup scheme, which consists in limiting the integral error to the supply voltage. This simple modification avoids controller overshoot in the event of output saturation. Figure 5 shows the normalized actuator step response for various amplitudes, which presents a 5\% response time of $100 \mathrm{~ms}$. Note that for a step larger than the design value of $60^{\circ}$, the response is no longer linear but does not present overshoot. Thanks to the chosen position sensor, the output fin position is accurate to $\pm 0.44^{\circ}$, with $0.078^{\circ}$ resolution and $0.027^{\circ}$ repeatability. The difference in time response among all four actuators is less than $10 \mathrm{~ms}$, which is negligible with respect to the airframe dynamics.

\section{B. Body Dynamics}

The projectile dynamics are subject to the flight mechanics principles [12], which lead to a nonlinear parameter-dependent model. In the present case, this model is simplified thanks to the absence of linear motion and the following assumed hypotheses for the study of the pitch axis: the airspeed $V$ is held constant and the other axes are locked at zero, therefore $p=0, r=0, \phi=0$ and $\psi=0$, where $\omega=\left[\begin{array}{lll}p & q & r\end{array}\right]^{\top}$ 


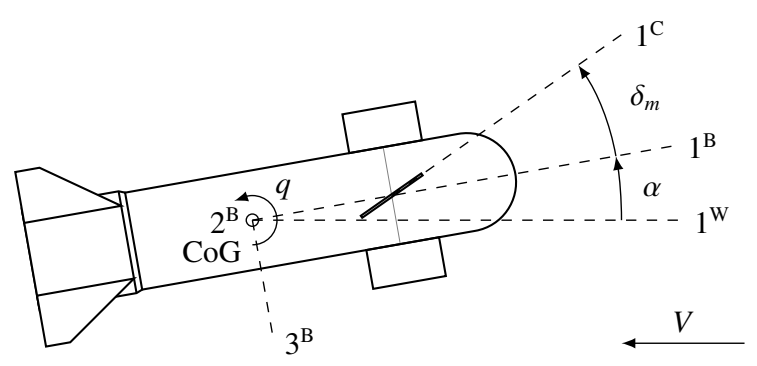

Fig. 6 Projectile side view and parameters.

is the body rate vector and $\phi$ and $\psi$ denote respectively the body roll and yaw angles. Furthermore, in this experimental setup the altitude $h$ is constant (ground level). Figure 6 presents the projectile in a pitching motion and the remaining parameters, which are the angle of attack $\alpha$, which is equal to the pitch angle $\theta$, the pitch rate $q$ and the control fin deflection $\delta_{m}$.

The pitch axis nonlinear model is linearized around a familiy of equilibrium (or trim) points, resulting in a second-order quasi-LPV model. In order to account for aerodynamic disturbances and measurement noise, a second-order disturbance model is appended to the projectile quasi-LPV model with the same poles. For any given operating point $\rho=\left[\begin{array}{ll}\bar{\alpha} & \bar{V}\end{array}\right]^{\top}$, this model can be written as:

$$
\begin{aligned}
& \dot{\boldsymbol{x}}_{\delta}(t)=\boldsymbol{A}(\boldsymbol{\rho}) \boldsymbol{x}_{\boldsymbol{\delta}}(t)+\boldsymbol{B}_{\boldsymbol{G}}(\boldsymbol{\rho}) \delta_{m, \delta}(t)+\boldsymbol{B}_{\boldsymbol{H}}(\boldsymbol{\rho}) d(t) \\
& y_{\delta}(t)=\boldsymbol{C}(\boldsymbol{\rho}) \boldsymbol{x}_{\boldsymbol{\delta}}(t)+\boldsymbol{D}_{\boldsymbol{G}}(\boldsymbol{\rho}) \delta_{m, \delta}(t)+\boldsymbol{D}_{\boldsymbol{H}}(\boldsymbol{\rho}) d(t)
\end{aligned}
$$

For simplicity, the $\delta$ subscript will be omitted for the rest of the article. The system matrices are:

$$
\left[\begin{array}{ccc}
\mathbf{A} & \mathbf{B}_{\mathbf{G}} & \mathbf{B}_{\mathbf{H}} \\
\mathbf{C} & \mathbf{D}_{\mathbf{G}} & \mathbf{D}_{\mathbf{H}}
\end{array}\right]=\left[\begin{array}{cc|c|c}
0 & 1 & 0 & M_{\alpha d} \\
M_{q \alpha} & M_{q q} & M_{q \delta} & M_{q d} \\
\hline 1 & 0 & 0 & 0 \\
0 & 1 & 0 & M_{\alpha d}
\end{array}\right]
$$

In the above matrices, the parameters $M_{q \alpha}, M_{q q}$ and $M_{q \delta}$ describe the dynamic behavior of the projectile and are related to the derivatives of the pitch aerodynamic coefficient $C_{m}$ at the considered operating point. Parameters $M_{\alpha d}$ and $M_{q d}$ describe the noise model and are scaled for a unity variance white noise signal $d(t)$. These parameters have been estimated for different values of the trim angle of attack $\bar{\alpha}$ from collected experimental data, using an estimation procedure based on numerical optimization [13] [14]. The estimated values of the parameters, and the corresponding uncertainty, are given in the appendix (Table 4). 
Although the values of the dynamic model parameters $\left(M_{q \alpha}, M_{q q}\right.$ and $\left.M_{q \delta}\right)$ are dependent on the trim angle of attack $\bar{\alpha}$, the maximum variation does not exceed $45 \%$ in the worst case. Furthermore, the corresponding second-order transfer function parameters (gain, corner frequency and damping) are shown in Table 5. The highest variation is on the damping $\zeta$, which varies from 0.21 at $\bar{\alpha}=0^{\circ}$ to 0.13 at $\bar{\alpha}=7^{\circ}$ $\left( \pm 21.6 \%\right.$ variation wrt the mean value), while the gain $k$ and corner frequency $\omega_{0}$ remain almost constant (less than $10 \%$ variation). Therefore, an adaptive or gain-scheduled controller may not be required for the constant speed case. The proposed approach consists in designing the autopilot for a nominal model, built using the mean estimated parameter values

$$
\begin{aligned}
\overline{\boldsymbol{\theta}} & =\left[\begin{array}{lllll}
M_{q \alpha} & M_{q q} & M_{q \delta} & M_{\alpha d} & M_{q d}
\end{array}\right]^{\top} \\
& =\left[\begin{array}{lllll}
-53.0712 & -2.4470 & 38.8179 & 0.0399 & 2.3232
\end{array}\right]^{\top}
\end{aligned}
$$

and in representing the deviations from this nominal model as uncertainty.

\section{Sensors}

The sensor model takes into account the bandwidth and noise contribution of the Inertial Measurement Unit. In this study, both effects are negligible and the IMU can be modeled by the unit transfer function: in terms of dynamics, the bandwidth of the sensors is much higher (over $200 \mathrm{~Hz}$ ) than the system open-loop bandwidth (less than $2 \mathrm{~Hz}$ ). The noise level has been assessed experimentally by comparing the variance of the pitch signal over a period of 10 seconds for two cases. In the first case, the projectile is at rest (zero airspeed), while in the second case the airspeed is raised to $V=25 \mathrm{~m} / \mathrm{s}$. As the variance in the latter case $\left(4.10^{-2}\right)$ is two orders of magnitude higher than the former $\left(3.10^{-4}\right)$, the noise contribution of the IMU can safely be neglected. Finally, a separate IMU model is neither necessary nor desirable since the body model is estimated from recorded IMU measurement, taking them into account the sensor dynamics.

\section{Autopilot Design}

This section details the design of an angle of attack autopilot using the nominal model postulated in Section III, which is used throughout the pitch flight envelope and at a constant airspeed. The design goal is to obtain a low-order fixed-structure controller in order to simplify implementation as well as to prepare the ground for a future airspeed scheduling, while maintaining high performance levels. 


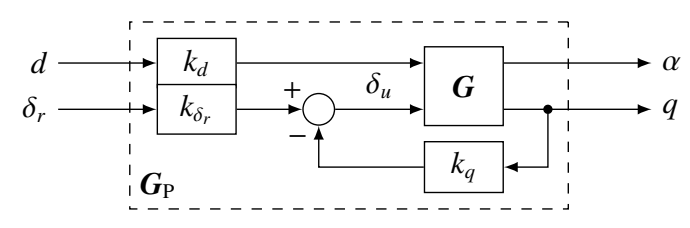

Fig. 7 Rate feedback loop, forming the damped open-loop plant $G_{\mathrm{P}}$.

\section{A. Objectives and Limitations}

The pitch axis autopilot aims at fulfilling several complementary objectives. The main goals are to augment the dynamic stability of the system and to track the reference AoA signal issued by guidance algorithms, while rejecting external disturbances such as wind gusts. The autopilot must also guarantee robust stability in the presence of uncertainty, such as neglected or unmodelled dynamics, or uncertainty on aerodynamic coefficients. Some of these goals are conflicting and lead the designer to a compromise, since improving performance degrades stability margins, and vice versa.

There are several limitations that must be taken care of during the design procedure. The resulting autopilot is to be implemented in Hardware-In-the-Loop experiments, where the control algorithms are executed on the embedded computer at an update rate $F_{s}=100 \mathrm{~Hz}$, and where the target software introduces a one-cycle controller delay [4]. The actuators have limited bandwidth and a limited saturation-free operating range as detailed in Section III. All these considerations limit the achievable closed-loop bandwidth and require the designer to keep a sufficient delay margin in the closed-loop autopilot system.

In the present case, the objective is to attenuate the effects of disturbances on the output $\alpha$ and provide accurate reference angle of attack tracking. The closed-loop system should behave like a second-order low-pass reference model $\boldsymbol{T}_{\text {ref }}$ with bandwidth $\omega_{0}=6 \mathrm{rad} / \mathrm{s}$ and damping $\zeta=0.78$. These performance requirements must not compromize stability, therefore the autopilot should comply with gain margin (GM) and phase margin (PM) requirements, where typical values in the aircraft and missile community are $\mathrm{GM}>6 \mathrm{~dB}$ and $\mathrm{PM}>45^{\circ}$.

\section{B. Rate Damping Controller}

One of the first functions of the autopilot is to improve the pitch plane dynamic stability of the projectile. In the field of missile and aircraft control design, this is accomplished by virtually increasing the aerodynamic damping derivative $M_{q q}$ through the use of a rate feedback loop [12], as presented in Fig. 7. 


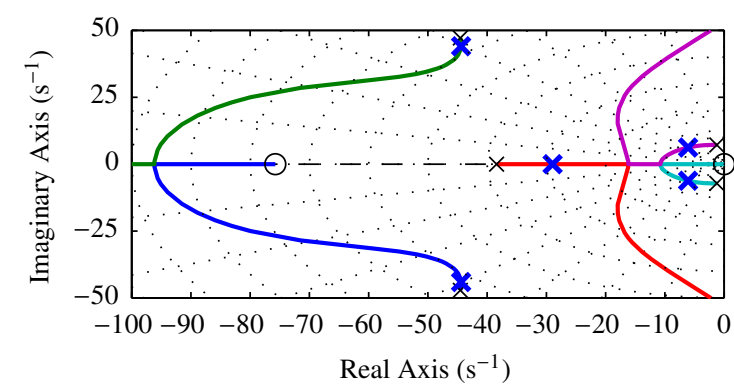

(a) Complete pole loci diagram

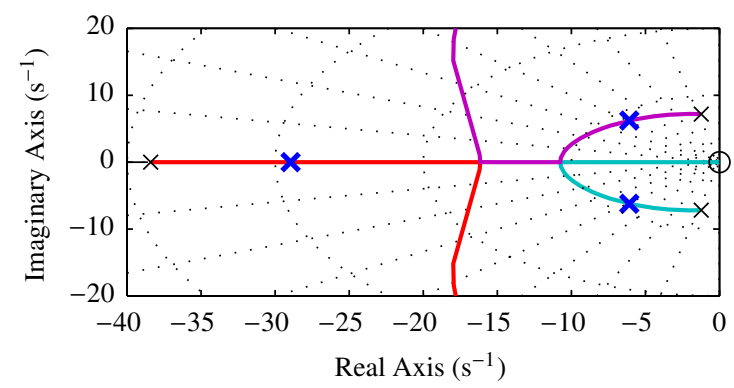

(b) Close-up on the low-frequency region, showing the improved

damping of the dominant poles

Fig. 8 Pole loci for the open-loop plant $G_{\mathrm{P}}$. Black crosses mark the location of open-loop $G$ plant poles. Bold blue crosses mark the location of rate damped plant poles.

In the present case, the open-loop nominal plant is largely underdamped with a damping ratio $\zeta_{G}=$ 0.17. Using a pole placement approach, the feedback gain $k_{q}$ is selected such that the closed-loop damping approaches 0.7 , while keeping the natural frequency of the actuator and body poles separated by a factor of at least three, as shown on Fig. 8. The virtual control input $\delta_{r}$ and disturbance input $d$ are then respectively scaled by the gains $k_{\delta_{r}}$ and $k_{d}$ such that the DC gain between these inputs and the angle of attack output is equal to unity. The resulting plant will be further referenced to as the damped open-loop plant $\boldsymbol{G}_{\mathrm{P}}$ and the variable to be controlled is the angle of attack $\alpha$. The obtained gain values for the rate damping controller are $k_{\delta_{r}}=1.538, k_{d}=19.49$ and $k_{q}=0.1914$

Figure 9 presents simulated and experimental responses of the closed loop against the open-loop response. Obviously, the rate damping controller can not constitute a tracking autopilot on its own, as any plant gain uncertainty will lead to a static error, and the system output is very sensitive to disturbances. Nevertheless, the system damping is vastly improved and there is an overall good agreement between the simulated and the experimentally observed responses. The difference between these two responses is caused by aerodynamic disturbances on the real system, which are not present in the simulation.

\section{Angle of Attack Autopilot}

The proposed angle of attack autopilot structure detailed throughout this section is illustrated in Fig. 10. It is composed of two separate controller blocks, resulting in a two-degrees of freedom control structure. The disturbance rejection feedback controller $\boldsymbol{K}_{\mathrm{FB}}$ acts on the tracking error signal $e=\alpha_{f}-\alpha$ and produces 


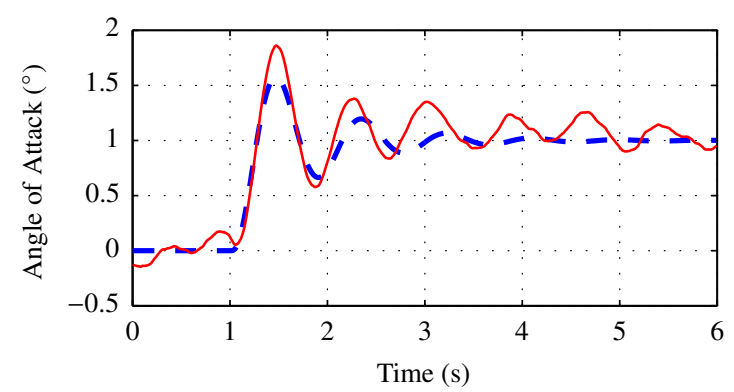

(a) Open-loop step response

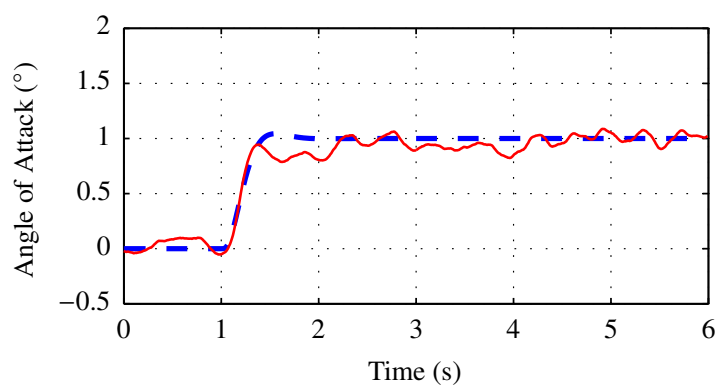

(b) Rate damping controller step response

Fig. 9 Open-loop and rate damping controller step responses. Dashed blue line: simulated response, solid red line: measured response.

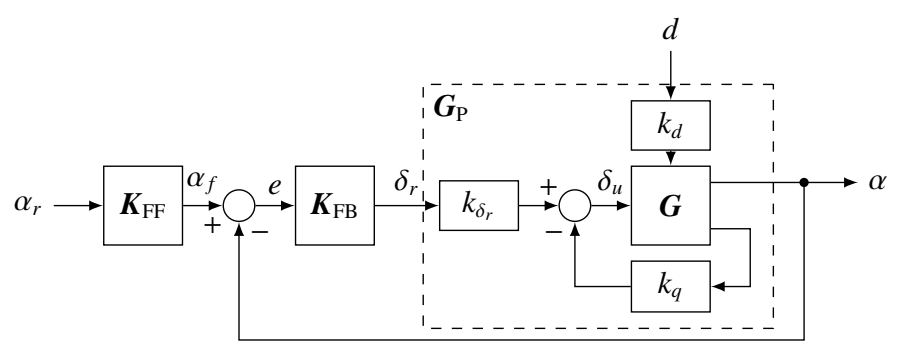

Fig. 10 Angle of attack autopilot structure.

the virtual control input $\delta_{r}$. The main role of $\boldsymbol{K}_{\mathrm{FB}}$ is to maintain closed-loop system stability and minimize the effects of the disturbance $d$ on the system outputs $\alpha$ and $q$. Proper reference tracking is ensured by the feedforward controller $\boldsymbol{K}_{\mathrm{FF}}$, which shapes the reference signal $\alpha_{r}$ such that the achieved angle of attack $\alpha$ follows the response of a reference model $\boldsymbol{T}_{\text {ref. }}$.

In the following paragraphs, the feedback controller is designed using two $\mathcal{H}_{\infty}$ approaches, leading respectively to a full-order controller and a fixed-order fixed-structure controller for comparison purposes. The feedforward controller is obtained first by closed-loop system inversion followed by a reduction step, and in a final step the design of both feedforward and feedback controllers is done simultaneously using a multi-objective approach.

\section{Disturbance Rejection Controller}

In the autopilot structure presented in Fig. 10, the feedback controller $\boldsymbol{K}_{\mathrm{FB}}$ is entirely responsible for rejection of external disturbances, as the feedforward controller $\boldsymbol{K}_{\mathrm{FF}}$ does not act on the tracking error. This paragraph focuses on the design of $\boldsymbol{K}_{\mathrm{FB}}$, using modern robust $\mathcal{H}_{\infty}$ control techniques in a S/KS mixed- 


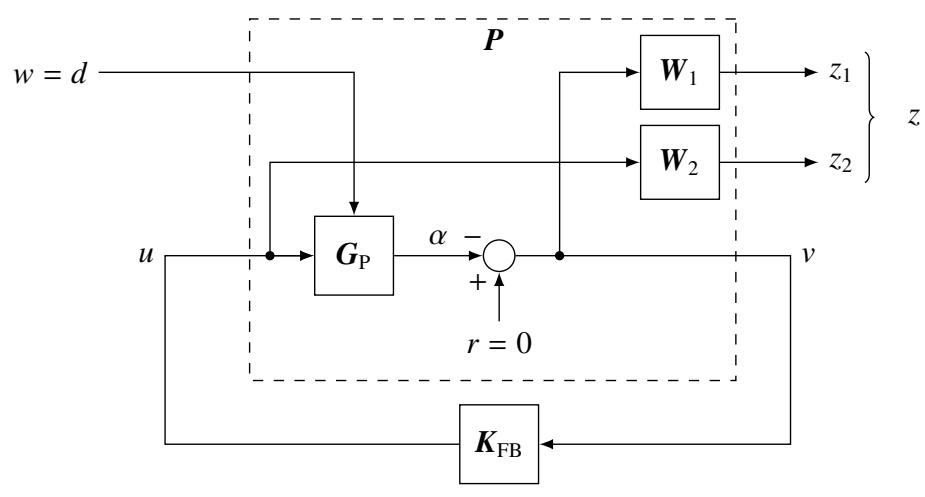

Fig. 11 Mixed S/KS sensitivity disturbance rejection problem in standard form.

sensitivity setup [15].

The $\mathcal{H}_{\infty}$ design problem is represented in the standard form interconnection, illustrated in Fig. 11. To this end, the design objectives and constraints are included in the augmented plant $\boldsymbol{P}$, of which the inputs are the exogenous signal $w=d$ (disturbance) and the control input $u=\delta_{r}$. The outputs of $\boldsymbol{P}$ are the performance signals $z=\left[\begin{array}{ll}z_{1} & z_{2}\end{array}\right]^{\top}$, which are to be minimized, and the measured variable $v=\alpha$. The $\mathcal{H}_{\infty}$ control problem then consists in finding $\boldsymbol{K}_{\mathrm{FB}}$, such that the system is nominally stable and

$$
\left\|\boldsymbol{T}_{w \rightarrow z}\right\|_{\infty}<\gamma, \quad \gamma>0
$$

where $\boldsymbol{T}_{w \rightarrow z}$ is the transfer function from $\boldsymbol{w}$ to $z$, which is obtained by the lower linear fractional transformation (LFT) $\boldsymbol{T}_{w \rightarrow z}=\mathcal{F}_{l}\left(\boldsymbol{P}, \boldsymbol{K}_{\mathrm{FB}}\right)$.

The S/KS mixed-sensitivity problem consists in shaping the closed-loop system sensitivity function $\boldsymbol{S}_{d \rightarrow e}$, which is the transfer function between the disturbance $d$ and the regulation error $e$, and $\boldsymbol{K}_{\mathbf{F B}} \boldsymbol{S}_{d \rightarrow e}$, which is the transfer function between the disturbance $d$ and the control input $u$ and corresponds to the control effort.

The body disturbance model $\boldsymbol{H}_{\mathrm{B}}$ acts as a low-pass filter on the disturbance signal $d$, which will be effectively rejected if the sensitivity function $S_{d \rightarrow e}$ presents small gain at low frequencies. Another requirement is to limit the controller effort, as the actuators have limited bandwidth and are prone to saturation in case of high-amplitude input signals. Therefore, the controller should limit both the amplitude and the bandwidth of the control signal $u$. These requirements are implemented using the weighting filters $\boldsymbol{W}_{1}$ and $\boldsymbol{W}_{2}$, which are here chosen as first-order systems of the form: 


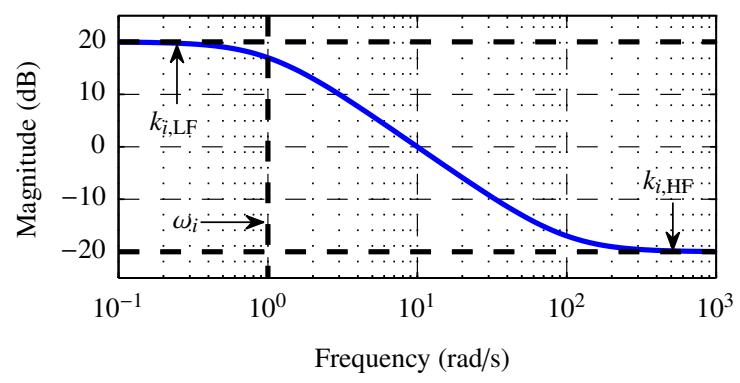

Fig. 12 Inverse of the weighting filters $W_{i}$ and associated parameters.

$$
\boldsymbol{W}_{i}=\frac{s+\omega_{i}}{k_{i, \mathrm{HF}} s+k_{i, \mathrm{LF}} \omega_{i}}
$$

The inverse of these weighting filters roughly correspond to the desired shape of the closed-loop transfer functions, and therefore the parameters specify the shape of the inversed filter, where $k_{i, \mathrm{HF}}$ is its gain at high frequencies, $k_{i, \mathrm{LF}}$ is its gain at low frequencies and $\omega_{i}$ its $-3 \mathrm{~dB}$ bandwidth frequency.

The parameters of the filter $\boldsymbol{W}_{1}$ acting on $\boldsymbol{S}_{d \rightarrow e}$ are selected such that its inverse exhibits a high-pass behavior. More specifically, the low-frequency gain $k_{1, \mathrm{LF}}$ must be low to reduce steady-state error but must be greater than zero in order to avoid numerical issues in the resolution of the $\mathcal{H}_{\infty}$ problem. The highfrequency gain $k_{1, \mathrm{HF}}$ and the bandwidth $\omega_{1}$ define the tolerated disturbance amplitude and bandwidth. The weighting filter $\boldsymbol{W}_{2}$ acts as a roll-off filter to limit the control gain after a certain frequency, which should be lower than the actuators bandwidth. The low-frequency gain $k_{2, \mathrm{LF}}$ must be greater than one, to allow controller action in the low frequencies, and the high-frequency gain $k_{2, \mathrm{HF}}$ is subject to the same numerical requirements as $k_{1, \mathrm{LF}}$. The selection of the weighting filter parameters is a process subject to trial-and-error, where the designer has to make a trade-off between effectiveness of disturbance rejection, control signal energy and open-loop stability margins. The finally selected filter parameters are summarized in Table 1, and the resulting performance transfer function $\boldsymbol{T}_{w \rightarrow z}$ is:

$$
\boldsymbol{T}_{w \rightarrow z}=\left[\begin{array}{c}
\boldsymbol{S}_{d \rightarrow e} \cdot \boldsymbol{W}_{1} \\
\boldsymbol{K}_{\mathrm{FB}} \cdot S_{d \rightarrow e} \cdot \boldsymbol{W}_{2}
\end{array}\right]
$$

As a first approach, the $\mathcal{H}_{\infty}$ problem of Eq. (5) is first solved using classical techniques (solving a Riccati equation[16] or an LMI formulation[17]), resulting in a full-order controller structure. The high-frequency modes are subsequently eliminated using model reduction techniques, and the controller is adjusted so that 
the lowest frequency pole is moved to zero, thereby eliminating steady-state error. In the end, a fifth-order controller $\boldsymbol{K}_{\mathrm{FB}, \mathrm{FO}}$ is obtained. However, full-order controllers are usually not suitable for implementation on low-power control electronics, and are difficult to integrate in a future gain-scheduling scheme. Here, this controller serves as a performance standard for comparison against reduced-complexity controllers.

In the second approach, a fixed-order, fixed-structure technique is employed, resulting in a reduced controller order and hence a much simpler structure, which can be imposed by the designer. In guided projectile control design, PI-type structures have been used successfully in stabilizing and reference tracking controllers [18]. The PID controller provides additional damping through the derivative term, however the derivative must be approximated using a low-pass filter such that the controller is proper and so as to limit the high-frequency gain. The fixed-structure controller $\boldsymbol{K}_{\mathrm{FB}, \mathrm{FS}}$ to be synthesized here is then of the form:

$$
\boldsymbol{K}_{\mathrm{FB}, \mathrm{FS}}=k_{p}+k_{i} \frac{1}{s}+\frac{k_{d} s}{T_{f} s+1}
$$

with parameters $k_{p}, k_{i}, k_{d}$, and $T_{f}$ to be determined. The $\mathcal{H}_{\infty}$ problem is then non-convex, and the nonsmooth $\mathcal{H}_{\infty}$ synthesis method of Apkarian and Noll [8] is employed to compute the fixed-order, fixedstructure controller $\boldsymbol{K}_{\mathrm{FB}, \mathrm{FS}}$.

\section{Reference Tracking Controller}

The above designed feedback controllers are focused on disturbance rejection. Even if the closed-loop system has no steady-state error, the time response does not conform to the specified requirements because the complete system should behave like the reference model $\boldsymbol{T}_{\text {ref. }}$. A straightforward approach for designing the feedforward filter $K_{\mathrm{FF}}$ consists in connecting the reference model and the inverse of the closed-loop transfer function $\boldsymbol{T}_{\alpha_{f} \rightarrow \alpha}$ in series [15]:

$$
K_{\mathrm{FF}}=\boldsymbol{T}_{\text {ref }} \boldsymbol{T}_{\alpha_{f} \rightarrow \alpha}^{-1}
$$

Obviously, the resulting system is non-proper and cannot be directly implemented on the real system. The approach employed to circumvent this limitation consists in approximating the magnitude frequency response of the ideal feedforward on a specific frequency band with a fixed-order transfer function. As the ideal feedforward filter behaves as a derivative filter at high frequencies, it is desirable to use a strictly proper 


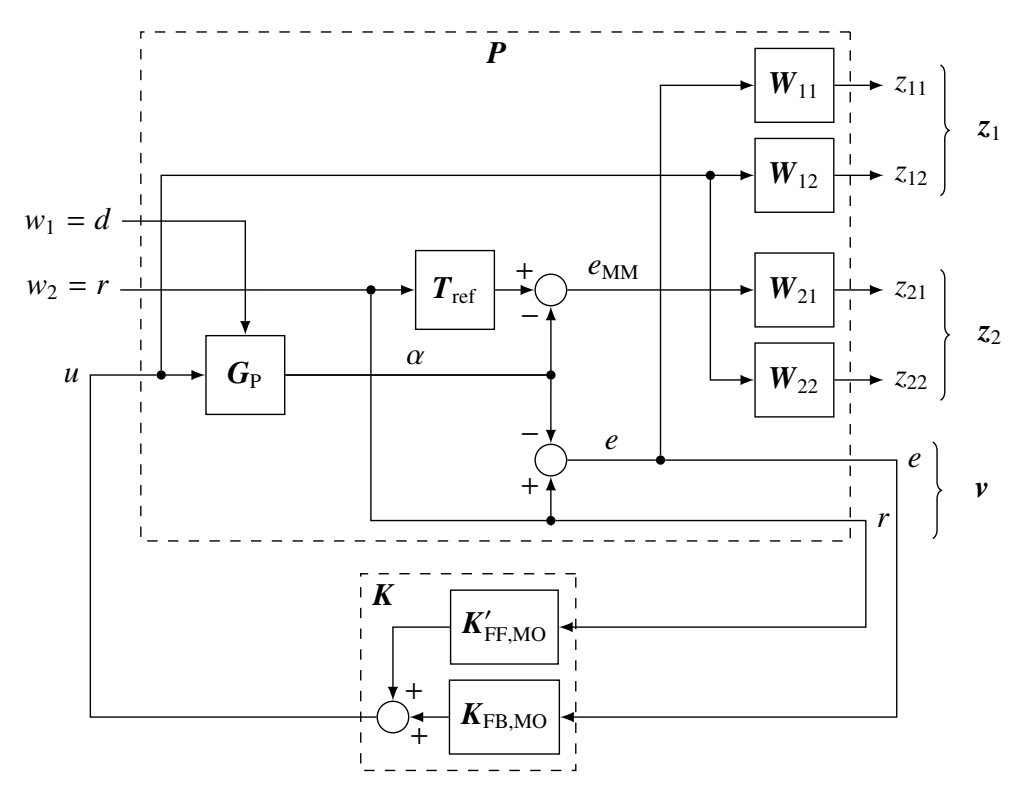

Fig. 13 Multi-objective two-degrees of freedom synthesis diagram in standard form.

transfer function (i.e. that has more poles than zeros), so as not to excite high-frequency dynamics of the closed-loop system. The frequency band must also be chosen with care as the high-frequency dynamics of the ideal feedforward filter may not be relevant and usually impose a higher-order transfer function and high gains. The selection of these parameters therefore results in a trade-off between model-matching accuracy (minimizing the difference between the reference model and the closed-loop behavior) and controller/actuator effort. However, this inverse-based design procedure may result in a too high model-matching error for low-order systems, and the designer's actions are quite limited. Moreover, while the disturbance rejection controller satisfies the constraints on the actuator control signal, the closed-loop system with feedforward may violate these specifications. It is more desirable to design the feedback and the feedforward controllers in a single step, resulting in a 2-DoF controller such that all constraints are applied during synthesis, as presented in the next subsection.

\section{Multi-objective Synthesis}

A naive approach to the design of the 2-DoF controller would be to add the feedforward controller and associated model-matching and controller effort constraints to the mixed-sensitivity problem of Fig. 11. The exogenous input vector $w$ then consists in the reference input $r$ as well as the disturbance input $v$. However, it is not possible to define the weighting filters $W_{i}$ for individual transfers, as all inputs are used during 
synthesis. For example, the performance signal relative to the model-matching error would include transfers from both inputs, when only the reference is relevant. This limits the designer's actions and leads to an unsatisfactory result.

As the simultaneous feedback and feedforward design problem addresses different objectives, a better formulation lies in multi-objective methods $[10,19]$. In these frameworks, the different requirements are specified in multiple $\boldsymbol{w}_{j} \rightarrow z_{j}$ channels for performance assessment, where the exogenous inputs $\boldsymbol{w}_{j}$ and performance outputs $z_{j}$ are vectors. The resulting $\boldsymbol{T}_{\boldsymbol{w}_{j} \rightarrow z_{j}}$ transfers specify these requirements independently of each other, as opposed to the case discussed previously. The synthesis procedure then consists in finding $\boldsymbol{K}$ such that the nominal system is stable and all requirements are satisfied simultaneously:

$$
\left\|\left\{\begin{array}{c}
\boldsymbol{T}_{\boldsymbol{w}_{1} \rightarrow z_{1}} \\
\boldsymbol{T}_{\boldsymbol{w}_{2} \rightarrow z_{2}} \\
\ldots \\
\ldots \\
\boldsymbol{T}_{\boldsymbol{w}_{j} \rightarrow z_{j}}
\end{array}\right)\right\|_{\infty}<\gamma, \quad \gamma>0
$$

In the present case, two $\boldsymbol{w}_{j} \rightarrow z_{j}$ channels are considered. The first channel, $w_{1} \rightarrow z_{1}$, deals with the disturbance rejection requirements, which were specified in Section IV C 1. The reference tracking requirements, namely a model-matching constraint with respect to $\boldsymbol{T}_{\text {ref }}$ and a high-frequency roll-off constraint on $\boldsymbol{K}_{\mathbf{F B}} S_{r \rightarrow e}$, which limits the injection of high-frequency dynamics in the plant from the reference input, are specified in the second channel $w_{2} \rightarrow z_{2}$, where $w_{2}=r$. These requirements are enforced by the means of the respective weighting filters $\boldsymbol{W}_{21}$ and $\boldsymbol{W}_{22}$. The model-matching filter $\boldsymbol{W}_{21}$ is a constant gain and acts on the model-matching error $e_{\mathrm{MM}}=r\left(\boldsymbol{T}_{\mathrm{ref}}-\boldsymbol{T}_{r \rightarrow \alpha}\right)$. The gain of $\boldsymbol{W}_{21}$ defines the maximum allowable difference between the target system and the closed-loop transfer function, which is the highest at medium frequencies. The roll-off filter $\boldsymbol{W}_{22}$ acts on the transfer $\boldsymbol{T}_{r \rightarrow u}$ and is specified in the same way as in the disturbance rejection case. However, the high-frequency limitation mostly impacts the feedforward filter and its bandwidth may be much larger than in the disturbance rejection case, as it will not compromize stability. The numerical values of the parameters of $\boldsymbol{W}_{21}$ and $\boldsymbol{W}_{22}$ are summarized in Table 1, and the performance transfer function 


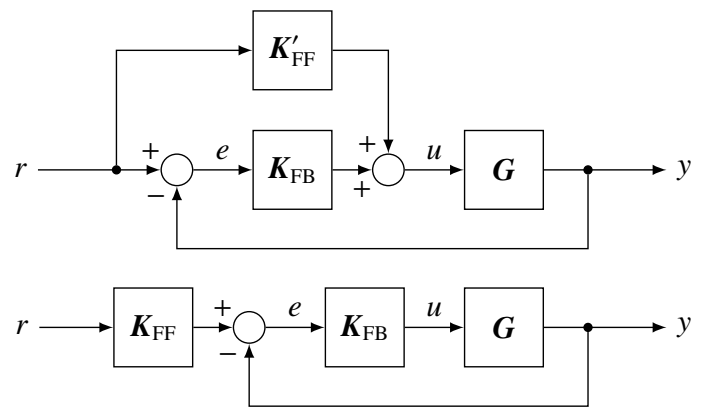

Fig. 14 Two-degrees of freedom control structures. Top: feedforward in series, or filter form. Bottom: feedforward in injection form.

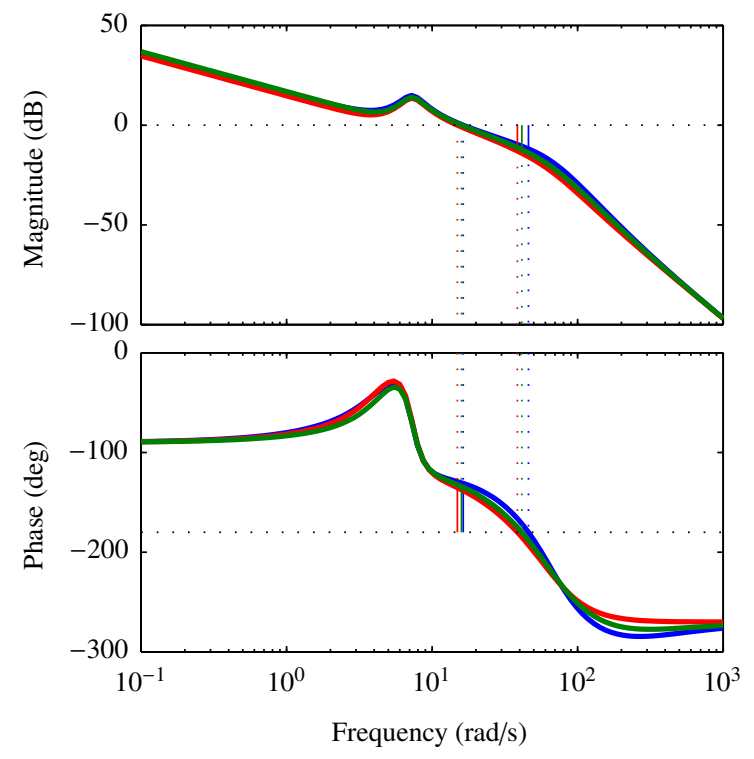

(a) Gain and phase margins, opening at the actuator input $\delta_{u}$

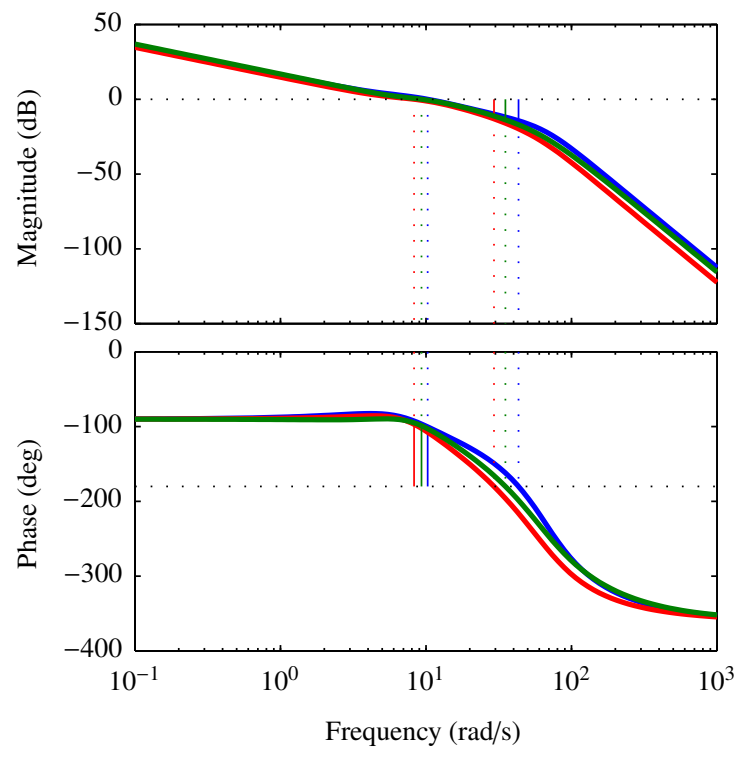

(b) Gain and phase margins, opening at the outer loop $\left(\delta_{r}, \boldsymbol{K}_{\mathrm{FB}}\right.$ output)

Fig. 15 Open-loop gain and phase margins for the disturbance rejection controllers (blue: full-order controller $\boldsymbol{K}_{\mathrm{FB}, \mathrm{FO}}$, red: fixed-structure controller $\boldsymbol{K}_{\mathrm{FB}, \mathrm{FS}}$, green:fixed-structure controller $\boldsymbol{K}_{\mathrm{FB}, \mathrm{MO}}$ in the multi-objective case).

$\boldsymbol{T}_{w \rightarrow z}$ is:

$$
\boldsymbol{T}_{w \rightarrow z}=\left[\begin{array}{cc}
\boldsymbol{S}_{d \rightarrow e} \cdot \boldsymbol{W}_{11} & 0 \\
\boldsymbol{K}_{\mathrm{FB}} \cdot S_{d \rightarrow e} \cdot \boldsymbol{W}_{12} & 0 \\
0 & \left(\boldsymbol{T}_{\mathrm{ref}}-\boldsymbol{T}_{\boldsymbol{r} \rightarrow \boldsymbol{\alpha}}\right) \cdot \boldsymbol{W}_{21} \\
0 & \boldsymbol{K}_{\mathrm{FB}} \cdot S_{r \rightarrow e} \cdot \boldsymbol{W}_{22}
\end{array}\right]
$$

The multi-objective synthesis problem of Eq. (10) is expressed in the standard form in Fig. 13 for the 


\begin{tabular}{|c|c|c|c|}
\hline \multicolumn{2}{|c|}{$\boldsymbol{W}_{1}=\boldsymbol{W}_{11}$} & \multicolumn{2}{|c|}{$\boldsymbol{W}_{2}=\boldsymbol{W}_{12}$} \\
\hline$k_{11, \mathrm{LF}}$ & 0.001 & $k_{12, \mathrm{LF}}$ & 1 \\
\hline$k_{11, \mathrm{HF}}$ & 0.6 & $k_{12, \mathrm{HF}}$ & 0.001 \\
\hline \multirow[t]{2}{*}{$\omega_{11}$} & $3 \mathrm{rad} / \mathrm{s}$ & $\omega_{12}$ & $15 \mathrm{rad} / \mathrm{s}$ \\
\hline & & & \\
\hline \multirow[t]{3}{*}{$k_{21}$} & 20 & $k_{22, \mathrm{LF}}$ & 1 \\
\hline & & $k_{22, \mathrm{HF}}$ & 0.001 \\
\hline & & $\omega_{22}$ & $150 \mathrm{rad} / \mathrm{s}$ \\
\hline
\end{tabular}

Table 2 Feedback controller synthesis results

\begin{tabular}{|c|c|c|c|}
\hline & Full-order & Fixed-structure & Multi-objective \\
\hline$\gamma$ & 1.26 & 1.38 & 1.36 \\
\hline Gain margin $^{a}$ & $14.40 \mathrm{~dB}$ & $13.08 \mathrm{~dB}$ & $13.69 \mathrm{~dB}$ \\
\hline Phase margin $^{a}$ & $79.50^{\circ}$ & $83.08^{\circ}$ & $81.22^{\circ}$ \\
\hline Gain margin ${ }^{b}$ & $11.88 \mathrm{~dB}$ & $12.93 \mathrm{~dB}$ & $12.22 \mathrm{~dB}$ \\
\hline Phase margin $^{b}$ & $49.01^{\circ}$ & $44.60^{\circ}$ & $45.79^{\circ}$ \\
\hline
\end{tabular}

${ }^{a}$ at the outer loop

${ }^{b}$ at the actuator input

two-channel case. The 2-DoF controller to be synthesized is composed of the feedback controller $\boldsymbol{K}_{\mathrm{FB}, \mathrm{MO}}$ and the feedforward controller $\boldsymbol{K}_{\mathrm{FF}, \mathrm{MO}}^{\prime}$, where the structure of $\boldsymbol{K}_{\mathrm{FB}, \mathrm{MO}}$ is defined in Eq. (8). In the first approach, the feedforward controller $\boldsymbol{K}_{\mathrm{FF}, \mathrm{MO}}$ is designed in filter-form, as in Fig. 10. However, an injection-form feedforward, as illustrated in Fig. 14, appears to achieve a similar level of performance with a lower-order structure and the design procedure behaves better numerically. In the end, the best performing structure is the first-order lead filter

$$
\boldsymbol{K}_{\mathrm{FF}, \mathrm{MO}}^{\prime}=k_{\mathrm{FF}, \mathrm{MO}} \frac{s+z_{\mathrm{FF}, \mathrm{MO}}}{s+p_{\mathrm{FF}, \mathrm{MO}}}
$$

of which an equivalent series form is given by:

$$
\boldsymbol{K}_{\mathrm{FF}, \mathrm{MO}}=1+\boldsymbol{K}_{\mathrm{FF}, \mathrm{MO}}^{\prime} \boldsymbol{K}_{\mathrm{FB}, \mathrm{MO}}^{-1}
$$




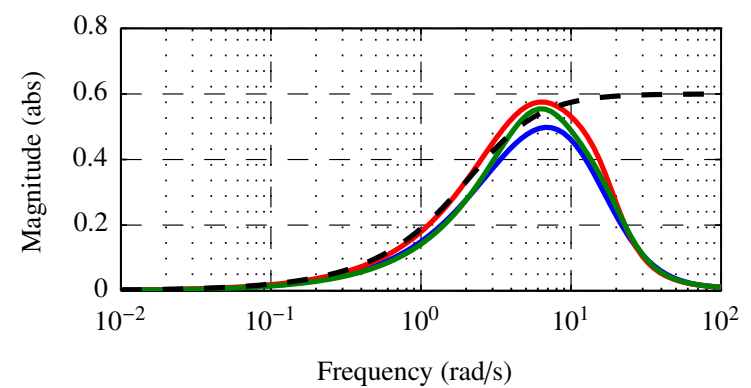

(a) Sensitivity transfers $\boldsymbol{S}_{d \rightarrow e}$ and constraint $1 / \boldsymbol{W}_{11}$

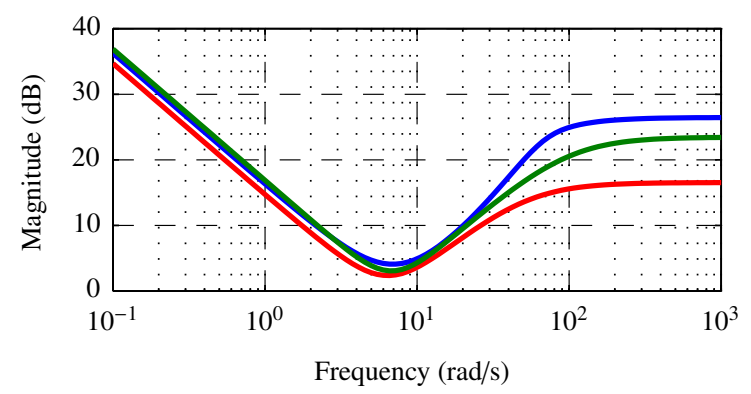

(c) Controllers Bode diagrams

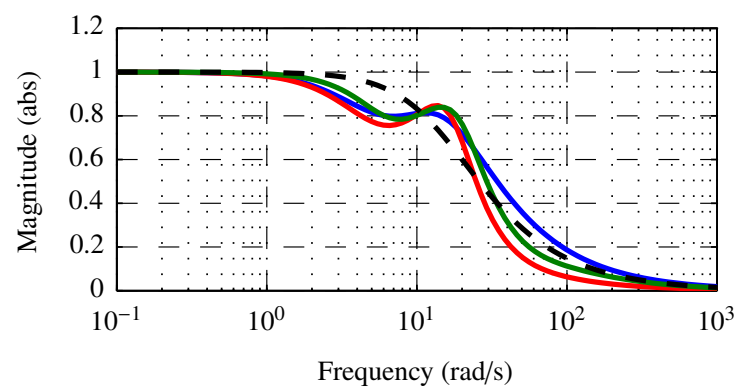

(b) Control sensitivity transfers $\boldsymbol{K}_{\mathbf{F B}} \boldsymbol{S}_{d \rightarrow e}$ and constraint $1 / \boldsymbol{W}_{12}$

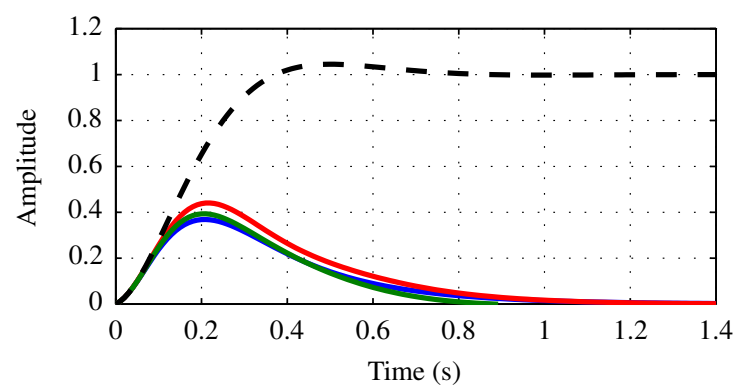

(d) Closed-loop response to a unit disturbance step

Fig. 16 Closed-loop transfer functions and unit step response for the disturbance rejection controllers (blue: fullorder controller $\boldsymbol{K}_{\mathrm{FB}, \mathrm{FO}}$, red: fixed-structure controller $\boldsymbol{K}_{\mathrm{FB}, \mathrm{FS}}$, green:fixed-structure controller $\boldsymbol{K}_{\mathrm{FB}, \mathrm{MO}}$ in the multiobjective case).

\section{Controller Synthesis and Simulation Results}

\section{Disturbance Rejection Controller}

The results of the feedback controller synthesis, using the three presented methods, are summarized in Table 2. In all cases, the minimum $\gamma$ is less than 1.4 , and the gain and phase margin requirements are met. The open-loop margins, shown in Fig. 15, have been computed for two loop opening sites: in the outer loop, at the output of $\boldsymbol{K}_{\mathrm{FB}}$ and in the inner rate damping loop, at the actuator input $\delta_{u}$.

The sensitivity transfer functions with respect to the disturbance input, as well as the controller Bode diagram and the closed-loop system response to a unit disturbance step are illustrated in Fig. 16. In these plots, the solid red and blue lines correspond respectively to the 1-DoF full-order and fixed-structure controllers $\boldsymbol{K}_{\mathrm{FB}, \mathrm{FO}}$ and $\boldsymbol{K}_{\mathrm{FB}, \mathrm{FS}}$, and the green line corresponds to the feedback controller $\boldsymbol{K}_{\mathrm{FB}, \mathrm{MO}}$ computed using the multi-objective procedure. In Fig. 16a and 16b, the dashed black line corresponds to the inverse of the weighting filters.

As specified in Section IV C, all feedback controllers were synthesized against the same requirements. 
The full-order controller $\boldsymbol{K}_{\mathrm{FB}, \mathrm{FO}}$ corresponds to the best achievable result, as there are no constraints on its structure. Nevertheless, it can be seen that both fixed-structure controllers exhibit similar performance, despite a less complex structure. As expected, the low-frequency error sensitivity $\boldsymbol{S}_{d \rightarrow e}$ tends to zero at low frequencies and does not exceed the upper limit specified in $\boldsymbol{W}_{11}$, and the control sensitivity $\boldsymbol{K}_{\mathbf{F B}} \boldsymbol{S}_{d \rightarrow e}$ tends to unity at low frequencies and zero at high frequencies. However, the controllers do not roll-off at high frequencies as the effect of the disturbance $d$ is already filtered out by the disturbance model $\boldsymbol{H}_{\mathrm{B}}$. Therefore, the spectral content of reference signal $r$ must be limited in order to limit the injection of high-frequency content in the feedback loop. This is guaranteed by the roll-off action of the feedforward controller.

Finally, Fig. 16d presents the closed-loop system response to a unity disturbance step against the openloop damped system, of which the response is represented by a black dashed line. The maximum deviation due to the disturbance is less than $0.45^{\circ}$, and the system reaches steady-state after about 1 second, with no static error.

\section{Reference Tracking}

The closed-loop transfer functions relative to the reference tracking objective, as well as the feedforward controller Bode diagrams and the autopilot step response, are illustrated in Fig. 17. These plots correspond to the before three considered controller structures: first the 1-DoF full-order and fixed-structure feedback controllers $\boldsymbol{K}_{\mathrm{FB}, \mathrm{FO}}$ and $\boldsymbol{K}_{\mathrm{FB}, \mathrm{FS}}$ with inverse-based feedforward controllers $\boldsymbol{K}_{\mathrm{FF}, \mathrm{FO}}$ and $\boldsymbol{K}_{\mathrm{FF}, \mathrm{FS}}$, respectively in solid blue and red, and second the fixed-structure feedback and feedforward controllers $\boldsymbol{K}_{\mathrm{FB}, \mathrm{MO}}$ and $\boldsymbol{K}_{\mathrm{FF}, \mathrm{MO}}$ computed using 2-DoF multi-objective synthesis in solid green.

In Fig. 17a and Fig. 17b, the model-matching error $e_{\mathrm{MM}}=\boldsymbol{T}_{\text {ref }}-\boldsymbol{T}_{r \rightarrow \alpha}$ and control sensitivity transfer $\boldsymbol{T}_{r \rightarrow u}$ are compared to the respective design constraints $1 / \boldsymbol{W}_{21}$ and $1 / \boldsymbol{W}_{22}$. In these figures, both inverse-based feedforward controllers violate the model-matching constraint but meet the control sensitivity requirement within a good margin. It must however be noted that these constraints are only effective during multiobjective synthesis, and the results obtained using inverse-based feedforward controllers are here presented only for comparison.

Figure 17c presents the Bode diagrams for the three feedforward controllers against the ideal, inversebased filters of Eq. (9), which are represented by dashed lines. In this figure, the equivalent series form of the 


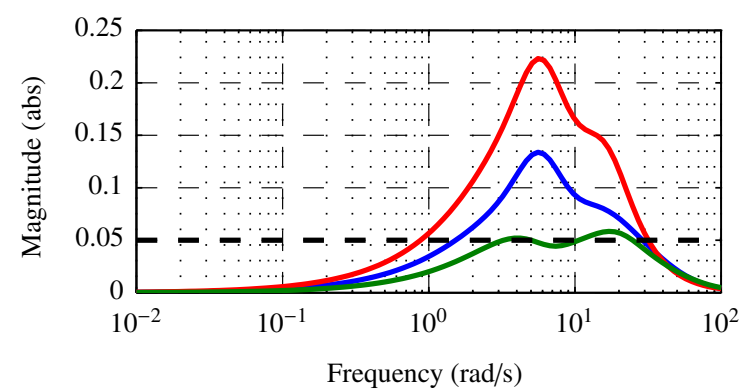

(a) Model-matching transfers $\boldsymbol{T}_{\text {ref }}-\boldsymbol{T}_{r \rightarrow \alpha}$ and constraint $1 / \boldsymbol{W}_{21}$

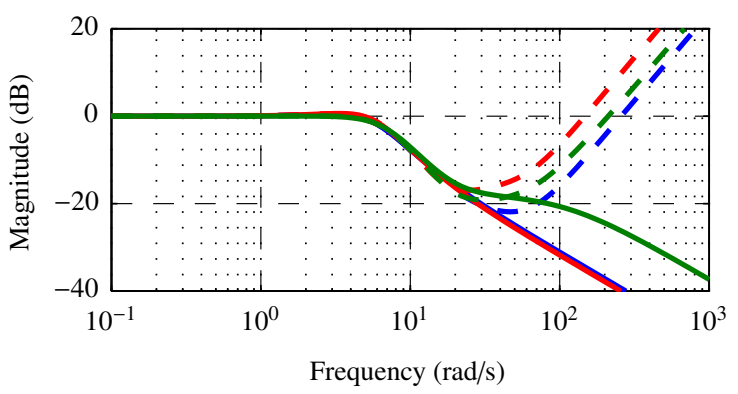

(c) Feedforward controllers Bode diagrams

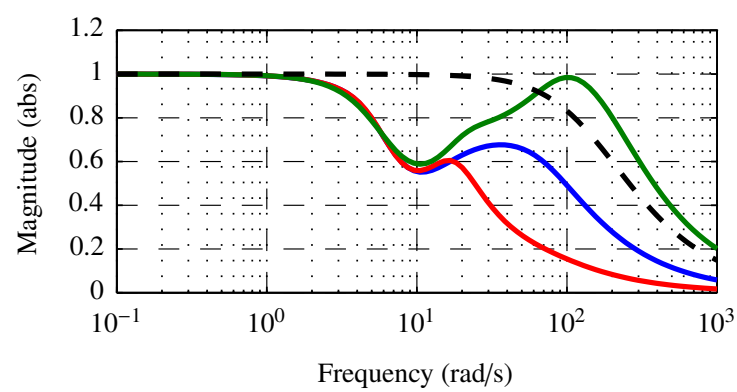

(b) Control sensitivity transfers $\boldsymbol{T}_{r \rightarrow u}$ and constraint $1 / \boldsymbol{W}_{22}$

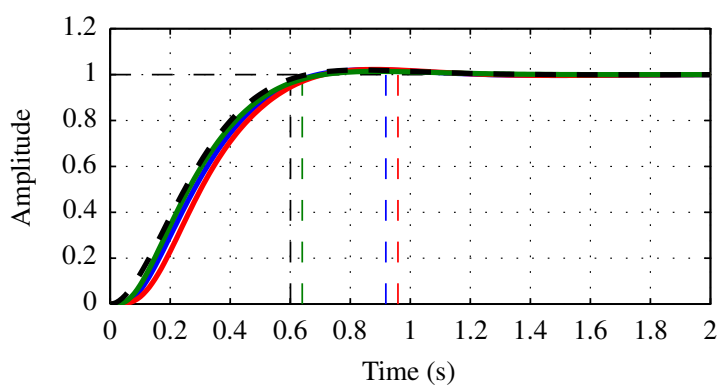

(d) Response to a unit reference step

Fig. 17 Closed-loop transfer functions and unit step response for the complete pitch axis autopilots (blue: fullorder controller $\boldsymbol{K}_{\mathrm{FB}, \mathrm{FO}}$ with inverse-based feedforward $\boldsymbol{K}_{\mathrm{FF}, \mathrm{FO}}$, red: fixed-structure controller $\boldsymbol{K}_{\mathrm{FB}, \mathrm{FS}}$ with inversebased feedforward $\boldsymbol{K}_{\mathrm{FF}, \mathrm{FS}}$, green:fixed-structure controller $\boldsymbol{K}_{\mathrm{FB}, \mathrm{MO}}$ and equivalent series feedforward controller $K_{\mathrm{FF}, \mathrm{MO}}$ designed using multi-objective synthesis).

injection feedforward controller $\boldsymbol{K}_{\mathrm{FF}, \mathrm{MO}}^{\prime}$ is used for comparison. As expected, all ideal inverse-based feedforward controllers have a similar shape, except in the high frequencies. The controllers obtained through approximation exhibit an identical response, while the multi-objective feedforward controller rolls off at higher frequencies, which is the best trade-off between the model-matching and the control sensitivity constraints respectively enforced with $\boldsymbol{W}_{21}$ and $\boldsymbol{W}_{22}$.

Finally, the time-domain performance is assessed in Fig. 17d by comparing the system response to a unit reference step against the response of the reference model $\boldsymbol{T}_{\text {ref }}$. Of the three designed autopilots, only the multi-objective controller follows the reference model specification, with a response time of $0.64 \mathrm{~ms}$. The two controllers obtained using the two-step approach achieve respective response times of $0.91 \mathrm{~ms}$ and $0.96 \mathrm{~s}$ for the full-order and the reduced-order controllers. In all three cases, the closed-loop response does not present steady-state error.

The inverse-based feedforward controllers $\boldsymbol{K}_{\mathrm{FF}, \mathrm{FO}}$ and $\boldsymbol{K}_{\mathrm{FF}, \mathrm{FS}}$ were designed according to Section IV C 2, 


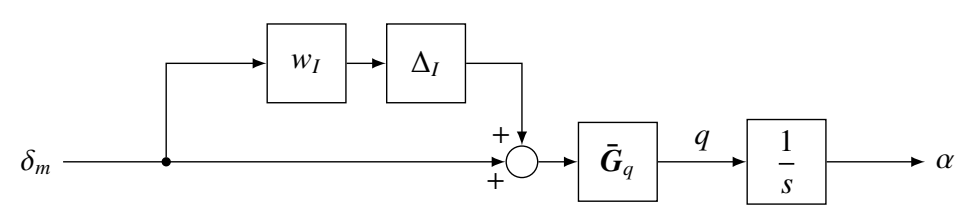

Fig. 18 Body dynamics model with multiplicative uncertainty.

using 2-pole, 1-zero transfer functions to approximate Eq. (9) for frequencies ranging from $0.1 \mathrm{rad} / \mathrm{s}$ to $10 \mathrm{rad} / \mathrm{s}$. As observed in Fig. 17c, the obtained filters fit the ideal inverse-based feedforward in the specified frequency band. These specifications were found using trial-and-error and result in a trade-off between controller complexity, model-matching error and controller effort. If a better conformance to the design constraints is required, the inverse-based design method is not as effective as multi-objective 2-DoF design, as in the latter case the requirements are taken into account during the synthesis of both controllers, while in the former case they are verified a posteriori.

\section{Robustness Analysis}

An important part of the $\mathcal{H}_{\infty}$ robust design process is to check whether the design specifications are respected even in the presence of uncertainty. This uncertainty in the plant may arise from many sources, such as neglected or unmodelled dynamics, uncertain parameters estimates or changes in operating conditions. To this end, the first step consists in modeling the uncertainty in the plant. In this section, the uncertain plant model is constructed using a multiplicative unstructured uncertainty representation, based on the difference between the nominal model and the family of observed models, as presented in Section III B. In a second step, the robust stability of the autopilot is assessed: the system must remain stable for all perturbations in the uncertainty set.

\section{A. Uncertainty Modeling}

The controllers were designed using a nominal "virtual" dynamics model based on mean estimated parameter values, as proposed in Section III B. In this paragraph, the proposed uncertain model takes into account the operating point variations as well as the uncertainty on estimated parameters.

In the body dynamics model of Eqs (2)-(3), the pitch rate $q$ is the derivative of the angle of attack $\alpha$. As the uncertain parameters only affect $q$, this model can be rewritten as a SISO uncertain pitch rate dynamics 


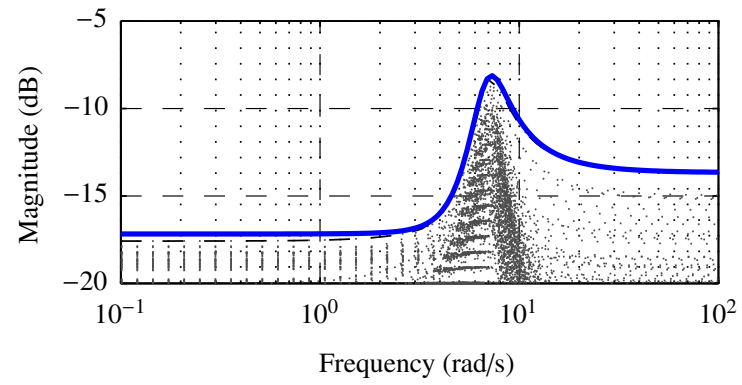

Fig. 19 Relative error for all observed realizations (dotted lines). Dashed line: uncertainty radius $\ell_{I}$. Solid line: second-order weight $w_{I}$ satisfying Eq. (16).

model $\boldsymbol{G}_{q}$, followed by an integrator, as illustrated in Fig. 18. A commonly used uncertainty representation is the multiplicative input uncertainty [15], where the perturbed plant form is:

$$
\boldsymbol{G}_{q}(s)=\overline{\boldsymbol{G}}_{q}(s)\left[1+w_{I}(s) \Delta_{I}(s)\right] \quad\left|\Delta_{I}(j \omega)\right| \leq 1, \forall \omega
$$

where $\overline{\boldsymbol{G}}_{q}$ is the nominal pitch rate dynamics model, $\Delta_{I}$ is any stable transfer function which is less than 1 in magnitude at each frequency. The multiplicative uncertainty weight $w_{I}$ is a minimum-phase rational transfer function that bounds the relative difference between the nominal plant and the set of all observed realizations. This set contains models estimated from experimental data as well as bootstrapped models, which were used to compute parameter uncertainties in Table 4. For each operating point, the bootstrapped models are estimated from fictive data, built from the nominal response and a random permutation of the model residues [20]. In the end, the set of observed models contains the 8 measured models and 30 bootstrapped models for each operating points, for a total of 248 models. At each frequency, the uncertainty radius $\ell_{I}(\omega)$ is:

$$
\ell_{I}=\max _{\boldsymbol{G}_{q}(s)}\left|\frac{\boldsymbol{G}_{q}(s)-\overline{\boldsymbol{G}}_{q}(s)}{\boldsymbol{G}_{q}(s)}\right|
$$

and the uncertainty weight $w_{I}$ satisfies

$$
\left|w_{I}(j \omega)\right| \geq \ell_{I}(\omega), \forall \omega
$$

In the present case, $w_{I}$ is selected as a second-order transfer function, i.e. the same order as the system. Figure 19 presents the obtained multiplicative weight against the relative error and uncertainty radius as per Eq. (15). 


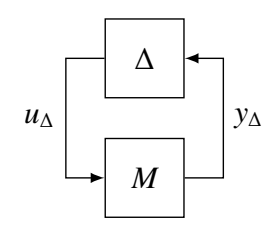

Fig. 20 Generic $M-\Delta$ structure for robust stability analysis.

\section{B. Robust Stability}

This subsection focuses on the stability of the proposed autopilots in the presence of uncertainty. To this end, the closed-loop plant is rewritten using the uncertain dynamics model given in Section V A. As the actual autopilots are to be implemented as a discrete-time control system, an additional 20 ms delay is inserted before the actuator input to account for the control computation delay and the zero-order hold, which arise from the discretization of the controllers.

In order to assess robust stability, the closed-loop system with uncertainty can be represented in the generic $M-\Delta$ structure of Fig. 20, where $M$ is the transfer function from the output to the input of the perturbation $\Delta_{I}$.

As the nominal system $M(s)$ and the perturbation $\Delta_{I}(s)$ are stable, the robust stability condition is, in the SISO case [15]

$$
|M(j \omega)|<1, \forall \omega
$$

which is a necessary and sufficient stability condition. The complete autopilot in $M-\Delta$ form is represented in Fig. 21, and the transfer function $M(s)$ for each feedback controller designed in Section IV is represented in Fig. 22. Note that the feedforward controller as well as the disturbance model are not included, as they are not part of any feedback loop. In all three cases, the closed-loop system is robustly stable to model uncertainty since the magnitude of the $M$ transfers remain under 1 at all frequencies. The overall autopilot is robustly stable even if the feedback controllers were designed without taking the actual control delay into account.

\section{Experimental Results}

This section deals with the validation of the multi-objective autopilot proposed in Section IV on the ACHILES test setup. The two other controllers will not be considered in the following paragraphs, as they 


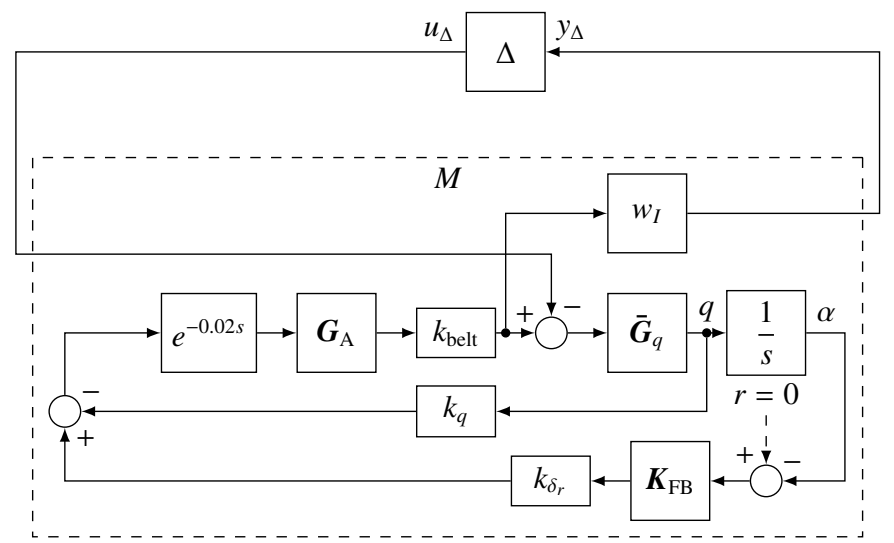

Fig. 21 Complete autopilot in $M-\Delta$ form.

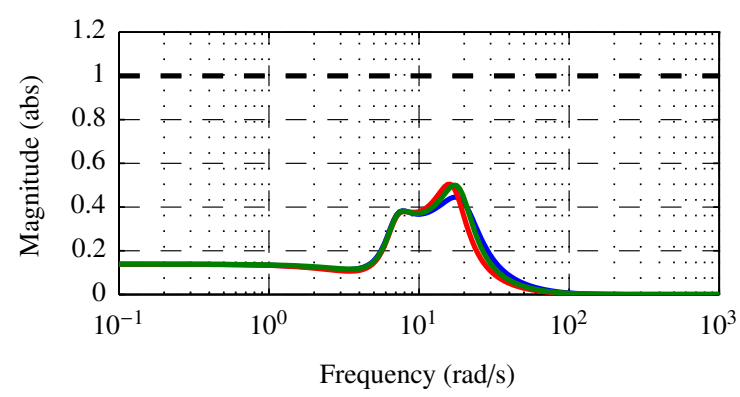

Fig. 22 Magnitude of the robust stability-related transfers $M$. Blue line: full-order controller $K_{\mathrm{FB}, \mathrm{FO}}$, red line: fixed-structure controller $\boldsymbol{K}_{\mathrm{FB}, \mathrm{FS}}$, green line: fixed-structure controller $\boldsymbol{K}_{\mathrm{FB}, \mathrm{MO}}$ designed using multi-objective synthesis. Black dashed line: stability bound on $M$.

exhibit higher complexity and lower performance than the multi-objective autopilot. The constraints related to the implementation on the embedded computer are first discussed, then the obtained experimental results are related to numerical simulation results and discussed.

\section{A. Hardware-In-the-Loop Implementation}

As presented in Section II, the ACHILES test setup constitutes a novel means of testing projectile control laws using real hardware in a restricted, controlled environment. In this setup, the projectile attitude and angular rates are measured by means of an IMU and the control algorithms are implemented on the embedded computer, both of which being discrete-time systems. In order to implement the above mentioned control laws, it is first necessary to convert them in an equivalent discrete-time representation.

The general form of the discrete-time autopilot is given in Fig. 23, where the controlled process $\boldsymbol{G}$ is a 


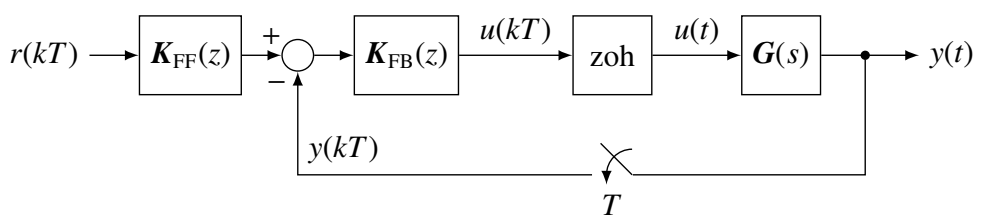

Fig. 23 General form of the discrete-time autopilot.

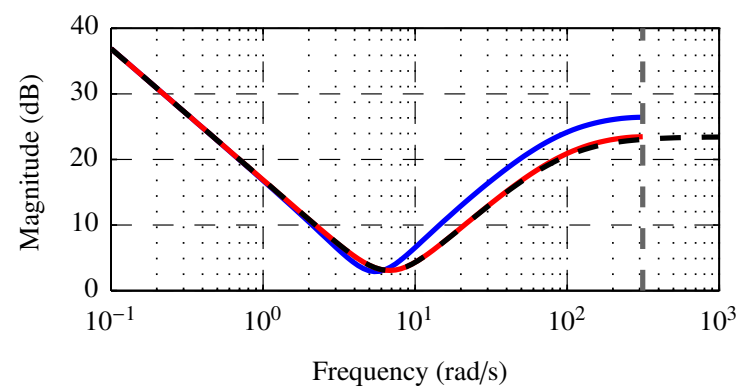

Fig. 24 Bode diagrams for comparison of discrete equivalents of $\boldsymbol{K}_{\mathrm{FB}}$ with $T=10 \mathrm{~ms}$. Blue line: using zero-order hold. Red line: using the bilinear transformation. Dashed black line: continuous-time controller

continuous-time system and the controller is executed in discrete timesteps. In the herein followed design by emulation method, the controller is first designed in the continuous domain, then discretized using an appropriate transform [21].

In the present case, the sampling period $T=10 \mathrm{~ms}$, and Fig. 24 compares the discrete equivalents of $\boldsymbol{K}_{\mathrm{FB}}$ obtained with a zero-order hold on the input and using the bilinear transformation. The best discretization is obtained by the bilinear transformation, as the former method is suited for a piecewise-constant input over a sampling period, which is not the case in a feedback control setup. The discretized controllers $\boldsymbol{K}_{\mathrm{FB}}, \boldsymbol{K}_{\mathrm{FF}}$ as well as the rate damping gain $k_{q}$ are then implemented in the Simulink diagram of Fig. 25, using the ACHILES custom blocks for interacting with the actuators and sensors.

\section{B. Results}

In Section IV, the three proposed autopilot structures have been designed using a "virtual" nominal model, based on the mean value of the estimated parameters. In this section, these autopilots have been implemented on the ACHILES test setup, as well as in the nonlinear simulator illustrated in Fig. 25c. This simulator is based on the nonlinear pitch axis model presented in Ref. [4] and which led to the $q$-LPV model of Eqs. (2)-(3). The dependency of the model parameters on the angle of attack is modeled using lookup 


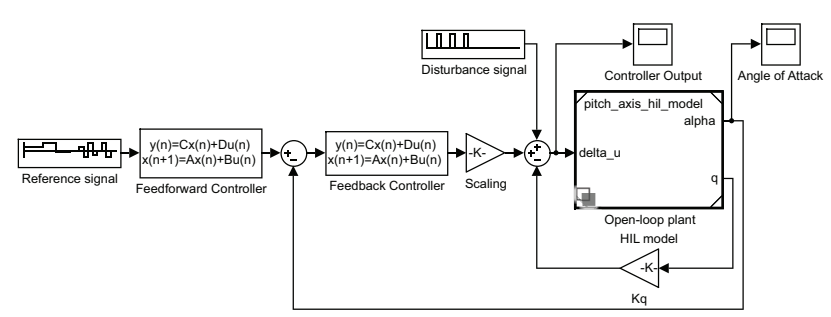

(a) High-level autopilot diagram

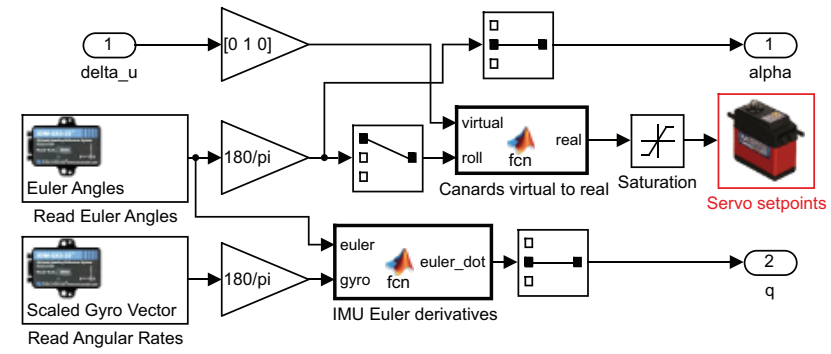

(b) Hardware-In-the-Loop implementation diagram

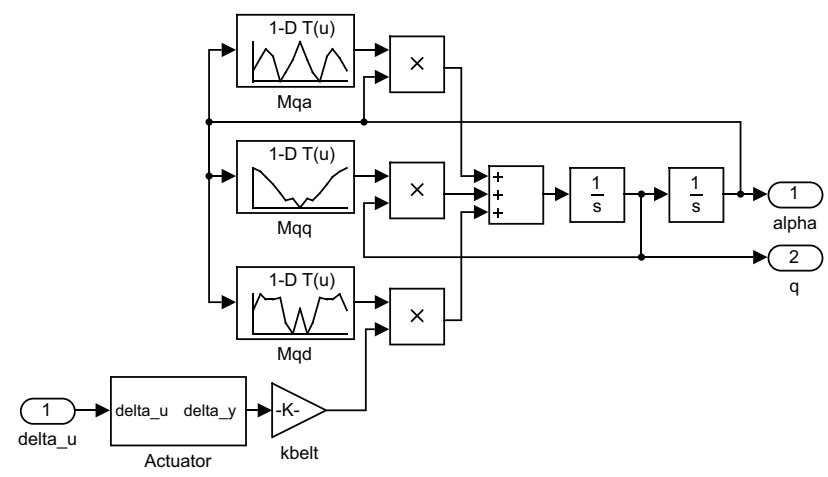

(c) Nonlinear simulation diagram

Fig. 25 Simulink diagrams used for autopilot validation. The "Open-loop plant" block in (a) references to diagram (b) for HIL experiments and to diagram (c) for numerical nonlinear simulations.

tables with the estimated parameter values of Table 4.

During the HIL experiments, the airspeed was set to the same value as for the system identification data collection experiments, i.e. $V=25 \mathrm{~m} / \mathrm{s}$.

\section{Disturbance Rejection Performance}

In the autopilot synthesis model, the disturbance $d$ is entering the open-loop system $\boldsymbol{G}$, where it is filtered by the disturbance model $\boldsymbol{H}_{\mathrm{B}}$. However, this disturbance signal cannot be influenced or measured directly in the case of HIL testing. As a result, in order to assess the disturbance rejection performance, an artificial disturbance step of known amplitude is injected at the plant input, i.e. at the $k_{q}$ summation point in Fig. 10, 


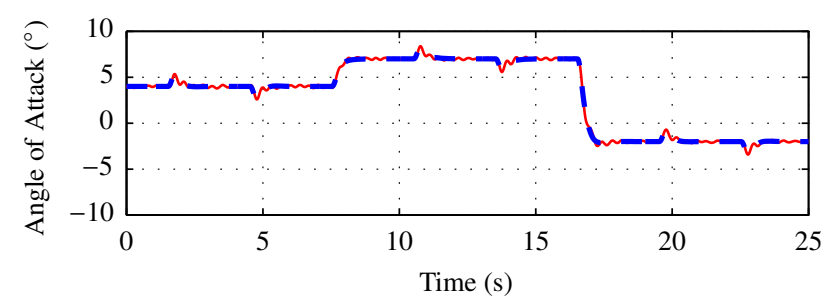

(a) Angle of Attack time response

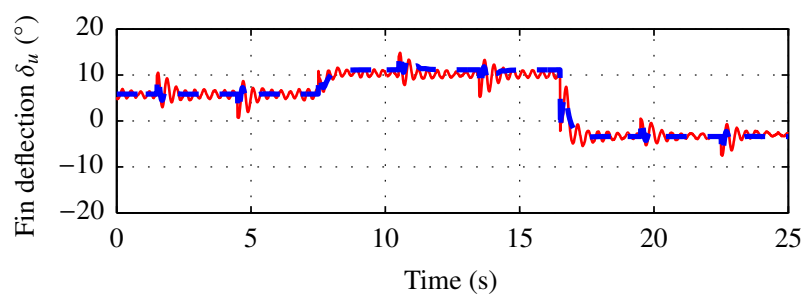

(b) Control signal $\delta_{u}$

Fig. 26 Disturbance rejection performance results, obtained with $4^{\circ}$ disturbance steps injected during steady-state.

Dashed blue line: nonlinear simulation. Solid red line: experimental results

for a constant reference angle of attack.

Figure 26 presents disturbance rejection results, where the $4^{\circ}$ disturbance step is injected while the system is at steady state. This is repeated for three different values of the reference angle of attack, so as to assess the effect of an operating point variation on the disturbance rejection performance.

In Fig. 26a, the observed response follows the simulation closely, and the disturbance is attenuated by more than $40 \%$ and rejected in less than 0.9 s. This is coherent with the results presented in Section IV and Fig. 17c. Figure $26 \mathrm{~b}$ presents the control signal $\delta_{u}$, where the peaks due to the injection of the disturbance are clearly visible. The steady-state difference between the simulated and the observed control signals is mainly due to uncertainty on the estimated DC gain, which does not impact performance thanks to the integrator in the feedback controller. As a final comment, both experimental signals exhibit visible superimposed oscillations. The exact source of these oscillations may emerge from an unmodeled nonlinearity (e.g. backlash in the servo-canard linkage), a flexible mode of the support system, interaction of the horizontal support rods and the tail fins, and/or to aerodynamic unsteadiness around the control surfaces and tail fins.

\section{Reference Tracking Performance}

The performance of the multi-objective autopilot is finally assessed and compared to the simulation using the nonlinear model, as well as the target reference model $\boldsymbol{T}_{\text {ref }}$. In the present case, the input signal is 


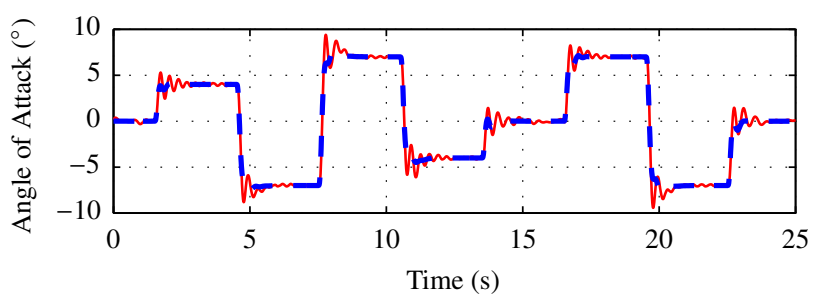

(a) Angle of Attack time response, without feedforward

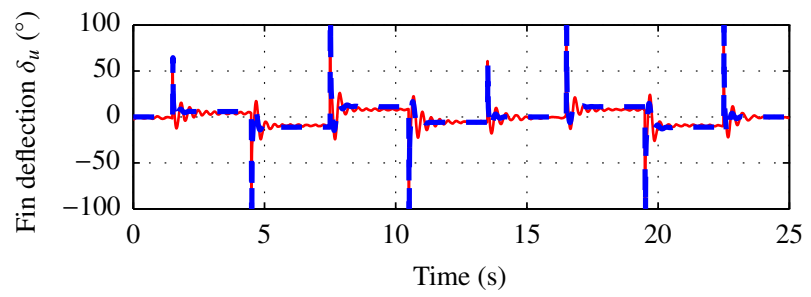

(b) Control signal $\delta_{u}$, without feedforward. The vertical scale has been

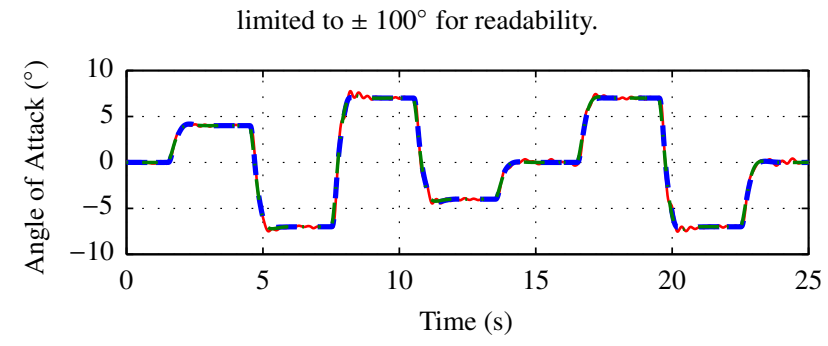

(c) Angle of Attack time response, with feedforward. Dashed-dotted green line is the response of the reference model $\boldsymbol{T}_{\text {ref }}$

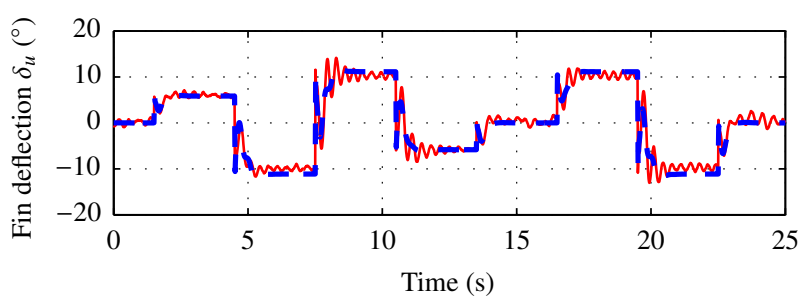

(d) Control signal $\delta_{u}$, with feedforward

Fig. 27 Full flight envelope reference tracking results. Dashed blue line: nonlinear simulation. Solid red line: experimental results.

a series of steps with different amplitudes, so as to exploit the whole projectile flight envelope.

Figure 27 presents the results of two reference tracking experiments using the multi-objective feedback controller with no feedforward controller in the first case (Figs. 27a and 27b), and with the associated feedforward filter in the second case (Figs. 27c and 27d).

In the latter case, the experimental and simulated responses are compared to the reference response using $\boldsymbol{T}_{\text {ref }}$, represented as a green dashed-dotted line in Fig. 27c. The three responses are almost undistinguishable, 
and the same level of performance as stated in Section IV is found. Figure $27 \mathrm{~d}$ presents the corresponding control signal $\delta_{u}$, which shows initial peaking on reference step, but with an amplitude not higher than the steady-state value.

In the absence of the feedforward filter, as highlighted by the former case, the reference signal no longer rolls off at high frequencies and the injection of these dynamics create visible overshooting and oscillations in the angle of attack output, as shown in Fig. 27a. Correspondingly, the control input $\delta_{u}$ exhibits considerable peaking, as illustrated in Fig. 27b. The amplitude and "sharpness" of these peaks exceed the actuator design constraints, resulting in actuator output saturation. Although not necessary for pure disturbance rejection, the feedforward controller is mandatory for respecting the reference tracking specifications.

\section{Conclusion}

In this article, a complete pitch axis autopilot design is carried out for the ACHILES projectile prototype. The ACHILES experimental setup is a novel benchmark for projectile control studies, where a projectile prototype is installed in a wind tunnel by the means of a 3-DoF gimbal. First, a plant model was built upon prior system identification studies, which yielded a family of linear models. The nominal synthesis model was then built using the mean values of estimated model parameters, and the difference modeled as multiplicative uncertainty.

In a second step, two autopilot design approaches have been considered. The considered autopilot structure consists in a feedback disturbance rejection controller and a feedforward reference tracking controller. In the first approach, these two controllers have been designed in separate steps, where the feedback controller was obtained using $\mathcal{H}_{\infty}$ synthesis techniques and the feedforward controller was designed using an inverse-based approximation. The second approach is based on recent multi-objective $\mathcal{H}_{\infty}$ synthesis methods, where both controllers are tuned in a single step. Compared to the first two-step approach, this latter technique yields fixed-structure controllers with slightly better performance and good conformance to the design specification, which greatly eases the designer's task.

As a robust control approach was followed for the autopilot design, the third step consisted in verifying the robust stability of the designed controllers. Using multiplicative unstructured uncertainty and an $M-\Delta$ representation, the three controllers were proved to be robustly stable against parameter uncertainty for the 
whole flight envelope.

The fourth and final step consisted in the validation of the multi-objective controller on the experimental setup and comparison against non-linear simulations, in order to assess the disturbance rejection and reference tracking performance. There is a very good agreement between the experimental and the simulation results, and both conform to the design specifications.

In future developments, the approaches proposed herein will be extended to integrate the projectile's yaw axis, in order to develop a coupled pitch-yaw autopilot.

\section{Appendix}

Table 3 Actuator model parameter values

\begin{tabular}{ccr}
\hline \hline Parameter & Value & Unit \\
\hline$K$ & 326.2 & $\%$ s/V \\
$\tau$ & 0.0182 & $\mathrm{~s}$ \\
$k_{p o s}$ & 29.4 & - \\
$k_{i}$ & 0.3065 & - \\
$k_{p}$ & 0.04 & - \\
\hline \hline
\end{tabular}

Table 4 Estimated parameters and associated uncertainty for values of $\bar{\alpha}$ from $0^{\circ}$ to $7^{\circ}$ in $1^{\circ}$ steps for a constant airspeed $V=25 \mathrm{~m} / \mathbf{s}$.

\begin{tabular}{cccccccccccc}
\hline \hline $\bar{\alpha}$ & $M_{q \alpha}$ & $\Delta M_{q \alpha}$ & $M_{q q}$ & $\Delta M_{q q}$ & $M_{q \delta}$ & $\Delta M_{q \delta}$ & $M_{\alpha d}$ & $\Delta M_{\alpha d}$ & $M_{q d}$ & $\Delta M_{q d}$ & Fit \\
\hline $0^{\circ}$ & -50.3 & $6 \%$ & -2.94 & $15 \%$ & 38.8 & $6 \%$ & 0.0511 & $2 \%$ & 3.16 & $3 \%$ & $74 \%$ \\
$1^{\circ}$ & -53 & $9 \%$ & -2.72 & $20 \%$ & 34.3 & $10 \%$ & 0.0421 & $2 \%$ & 3.19 & $3 \%$ & $75 \%$ \\
$2^{\circ}$ & -54.6 & $7 \%$ & -2.78 & $16 \%$ & 36.2 & $7 \%$ & 0.0394 & $2 \%$ & 2.87 & $5 \%$ & $82 \%$ \\
$3^{\circ}$ & -56.1 & $2 \%$ & -2.6 & $8 \%$ & 40.8 & $3 \%$ & 0.0591 & $2 \%$ & 2.14 & $5 \%$ & $86 \%$ \\
$4^{\circ}$ & -52.5 & $4 \%$ & -2.37 & $12 \%$ & 40.5 & $4 \%$ & 0.0431 & $2 \%$ & 2.29 & $5 \%$ & $89 \%$ \\
$5^{\circ}$ & -51.5 & $5 \%$ & -2.23 & $15 \%$ & 40.3 & $5 \%$ & 0.0387 & $2 \%$ & 2.38 & $3 \%$ & $89 \%$ \\
$6^{\circ}$ & -52.7 & $3 \%$ & -2.09 & $17 \%$ & 41.5 & $4 \%$ & 0.0416 & $2 \%$ & 2.52 & $3 \%$ & $89 \%$ \\
$7^{\circ}$ & -54.4 & $3 \%$ & -1.99 & $8 \%$ & 38.5 & $3 \%$ & 0.0528 & $1 \%$ & 2.35 & $5 \%$ & $86 \%$ \\
\hline \hline
\end{tabular}


Table 5 Airframe model gain $k$, corner frequency $\omega_{0}$ and damping ratio $\zeta$ for values of $\bar{\alpha}$ from $0^{\circ}$ to $7^{\circ}$ in $1^{\circ}$ steps for a constant airspeed $V=25 \mathrm{~m} / \mathrm{s}$.

\begin{tabular}{|c|c|c|c|c|c|c|c|c|c|}
\hline $\operatorname{Trim} \alpha$ & $0^{\circ}$ & $1^{\circ}$ & $2^{\circ}$ & $3^{\circ}$ & $4^{\circ}$ & $5^{\circ}$ & $6^{\circ}$ & $7^{\circ}$ & Variation \\
\hline$k$ & 0.77 & 0.65 & 0.66 & 0.73 & 0.77 & 0.78 & 0.79 & 0.71 & $\pm 9.58 \%$ \\
\hline$\omega_{0}(\mathrm{rad} / \mathrm{s})$ & 7.09 & 7.28 & 7.39 & 7.49 & 7.25 & 7.17 & 7.26 & 7.37 & $\pm 2.74 \%$ \\
\hline$\zeta$ & 0.21 & 0.19 & 0.19 & 0.17 & 0.16 & 0.16 & 0.14 & 0.13 & $\pm 21.58 \%$ \\
\hline
\end{tabular}

\section{Acknowledgments}

The authors wish to thank the French Ministry of Defence Procurement Agency (DGA, Direction Générale de l'Armement) for its financial support.

The authors also wish to thank Denis Bidino, Kevin Meder and all the staff at the ISL workshop for the mechanical design and manufacturing of the ACHILES test setup, along with Emmanuel Roussel and Joseph Juncker for their precious help during the development of the embedded electronics and software.

\section{References}

[1] Hann, C., Rao, A., Snowdon, M., Wongvanich, N., Winn, O., and Chen, X., "Minimal modeling of rocket roll response and aerodynamic disturbance in wind tunnel," IEEE International Conference on Control and Automation, 2011, pp. 213-218.

[2] Fresconi, F., Celmins, I., Ilg, M., and Maley, J., "Projectile Roll Dynamics and Control with a Low-Cost Maneuver System," Journal of Spacecraft and Rockets, Vol. 51, No. 2, March 2014, pp. 624-627.

[3] Patel, M. P., Tilmann, C. P., and Ng, T. T., “Closed-Loop Missile Yaw Control via Manipulation of Forebody Flow Asymmetrics," Journal of Spacecraft and Rockets, Vol. 41, No. 3, 2004, pp. 436-443.

[4] Strub, G., Gassmann, V., Theodoulis, S., Dobre, S., and Basset, M., "Hardware-In-the-Loop Experimental Setup Development for a Guided Projectile in a Wind Tunnel," 2014 IEEE/ASME International Conference on Advanced Intelligent Mechatronics (AIM), July 2014, pp. 458-463.

[5] Nichols, R., Reichert, R., and Rugh, W., "Gain scheduling for $\mathcal{H}_{\infty}$ controllers: a flight control example," IEEE Transactions on Control Systems Technology, Vol. 1, No. 2, 1993, pp. 69-79.

[6] Apkarian, P., Biannic, J., and Gahinet, P., "Self-scheduled $\mathcal{H}_{\infty}$ control of missile via linear matrix inequalities," Journal of Guidance, Control, and Dynamics, Vol. 18, No. 3, 1995, pp. 532-538.

[7] Theodoulis, S. and Duc, G., "Missile autopilot design: gain-scheduling and the gap metric," Journal of guidance, control, and dynamics, Vol. 32, No. 3, 2009, pp. 986-996. 
[8] Apkarian, P. and Noll, D., "Nonsmooth $\mathcal{H}_{\infty}$ Synthesis," IEEE Transactions on Automatic Control, Vol. 51, No. 1, 2006, pp. $71-86$.

[9] Gumussoy, S., Henrion, D., Millstone, M., and Overton, M. L., "Multiobjective Robust Control with HIFOO 2.0," Israel, 2009, pp. 144-149, arXiv: 0905.3229.

[10] Apkarian, P., Gahinet, P., and Buhr, C., "Multi-Model, Multi-Objective Tuning of Fixed-Structure Controllers," 2014 European Control Conference (ECC), IEEE, 2014, p. 856-861.

[11] Strub, G., Theodoulis, S., Gassmann, V., Dobre, S., and Basset, M., "Pitch Axis Control for a Guided Projectile in a Wind Tunnel-based Hardware-In-the-Loop Setup," AIAA Modeling and Simulation Technologies Conference, 2015.

[12] Zipfel, P. H., Modeling and Simulation of Aerospace Vehicle Dynamics, American Institute of Aeronautics and Astronautics, 2007.

[13] Strub, G., Gassmann, V., Theodoulis, S., Dobre, S., and Basset, M., "Pitch Axis Identification for a Guided Projectile using a Wind Tunnel-based Experimental Setup," Manuscript submitted for publication.

[14] Ljung, L., System Identification - Theory for the User, Prentice-Hall, 1999.

[15] Skogestad, S. and Postlethwaite, I., Multivariable Feedback Control: Analysis and Design, Second Edition, Vol. 2, John Wiley \& Sons, 2007.

[16] Doyle, J., Glover, K., Khargonekar, P., and Francis, B., "State-space solutions to standard $\mathcal{H}_{2}$ and $\mathcal{H}_{\infty}$ control problems," IEEE Transactions on Automatic Control, Vol. 34, No. 8, 1989, pp. 831-847.

[17] Gahinet, P. and Apkarian, P., "A linear matrix inequality approach to $\mathcal{H}_{\infty}$ control," International Journal of Robust and Nonlinear Control, Vol. 4, No. 4, 1994, pp. 421-448.

[18] Sève, F., Theodoulis, S., Wernert, P., Zasadzinski, M., and Boutayeb, M., "Pitch/yaw channels control design for a $155 \mathrm{~mm}$ projectile with rotating canards, using a $\mathcal{H}_{\infty}$ loop-shaping design procedure," AIAA Guidance, Navigation, and Control Conference, 2014.

[19] Scherer, C., Gahinet, P., and Chilali, M., "Multiobjective output-feedback control via LMI optimization," IEEE Transactions on Automatic Control, Vol. 42, No. 7, 1997, pp. 896-911.

[20] Walter, E. and Pronzato, L., Identification of Parametric Models from Experimental Data, Springer, 1997.

[21] Stevens, B. L. and Lewis, F. L., Aircraft Control and Simulation, John Wiley \& Sons, 2003. 